This is the author's final, peer-reviewed manuscript as accepted for publication. The publisher-formatted version may be available through the publisher's web site or your institution's library.

\title{
Strain Localization in Unsaturated Elastic-Plastic Materials Subjected to Plane Strain Compression
}

Dunja Peric, Gaofeng Zhao and Nasser Khalili

\section{How to cite this manuscript}

If you make reference to this version of the manuscript, use the following information:

Khalili, N., Zhao, G., \& Peric, D. (2014). Strain localization in unsaturated elastic-plastic materials subjected to plane strain compression.

\section{Published Version Information}

Citation: Khalili, N., Zhao, G., \& Peric, D. (2014). Strain localization in unsaturated elastic-plastic materials subjected to plane strain compression. Journal of Engineering Mechanics, 140(7), 04014050.

Digital Object Identifier (DOI): 10.1061/(ASCE)EM.1943-7889.0000767

Publisher's Link: http://ascelibrary.org/doi/abs/10.1061/\%28ASCE\%29EM.1943$\underline{7889.0000767}$

This item was retrieved from the K-State Research Exchange (K-REx), the institutional repository of Kansas State University. K-REx is available at http://krex.ksu.edu 


\title{
Strain Localization in Unsaturated Elastic-Plastic Materials Subjected to Plane Strain Compression
}

\author{
Dunja Perić*, A.M. ASCE, Gaofeng Zhao ${ }^{\dagger}$, and Nasser Khalili
}

\footnotetext{
* Associate Professor, Department of Civil Engineering, Kansas State University, Manhattan, KS 66506-5000, U.S.A.Email: peric@ksu.edu

${ }^{\dagger}$ Lecturer, School of Civil and Environmental Engineering, The University of New South Wales, Sydney NSW 2052, Australia Email:gaofeng.zhao@unsw.edu.au

${ }^{\ddagger}$ Professor and Associate Dean for Research, School of Civil and Environmental Engineering, The University of New South Wales, Sydney NSW 2052, AustraliaEmail:n.khalili@unsw.edu.au
} 


\begin{abstract}
Analytical solutions have been derived for the onset of strain localization in a broad class of unsaturated elastic-plastic porous materials. To this end, the definition of effective stress proposed by Khalili and Khabbaz (1998), which is based on a substantial amount of experimental evidence, has been adopted. Critical hardening moduli for constant water content and drained loadings were found to be further gradual simplifications of the critical hardening modulus for an undrained loading. In addition, the solutions were found to reduce to the previously found solutions for fully saturated and monophasic porous materials by adequately adjusting the bulk moduli of the pore fluids. This finding demonstrates that the mechanics of fully saturated and monophasic soils is the special simpler case of the mechanics of unsaturated soils. A diagnostic tool for detection of the inception of strain localization was developed by implementing the above solutions into a constitutive driver for a bounding surface plasticity model. The tool was used to further illustrate the strain localization behavior of unsaturated Bourke silt from Bourke region of New South Wales, Australia subjected to undrained, constant water content and drained loadings.
\end{abstract}

\title{
Subject headings: Bifurcations, Elastoplasticity, Silts, Unsaturated soils
}




\section{INTRODUCTION}

The onset of strain localization has been mathematically described as a bifurcation of the incremental solution for elastic-plastic solids such as metals and geological materials. Physically, it signifies the inception of narrow zones, typically orders of magnitude smaller than the underlying macro-scale problem, within which large strains evolve with continuing loading. These zones are commonly known as deformation bands and include shear, compaction and dilation bands, as well as combined shear and compaction or shear and dilation bands (Bésuelle 2001). The inception of deformation bands is a failure precursor because it signifies the initiation of an emerging localized failure mechanism. A majority of the vast amount of research that has been devoted to strain localization, particularly in the last three decades, has focused on monophasic materials. Significantly smaller amount of research has addressed two-phase materials while an extremely scarce amount has covered three-phase or unsaturated porous materials.

In various macro-scale boundary value problems such as laboratory soils samples and relevant in situ geomechanics situations the inception of strain localization is affected by boundary conditions, material inhomogeneities and stress states. For example in laboratory soil samples the onset of strain localization depends on the interplay of the sample inhomogeneity, the amount of friction imposed by the end platens, a height to diameter ratio, and the stress state or loading mode of the sample (Bigoni \& Hueckel 1991a,b; Perić et al. 1992,1993). A faithful computational characterization of material inhomogeneities remains elusive primarily due to our current inability to detect them reliably. Meanwhile, a diagnostic strain localization analysis is powerful tool that can provide an insight into the effects of stress state and drainage conditions on the onset of strain localization in unsaturated soils. 
The existing analytical solutions for the onset of strain localization range from those addressing one phase materials (Rudnicki and Rice 1975; Ottosen and Runesson 1991; Runesson et al. 1991) to two phase saturated materials (Runesson et al. 1996; Benallal and Comi 2002). Vardoulakis (1996a) and Vardoulakis (1996b) analyzed actual undrained plane strain experiments by using the framework of continuum mixtures theory for elastic-plastic materials with internal friction and dilatancy. He found that the initial homogenous deformation of saturated sand samples was followed by a spontaneous deformation of a persistent shear band. Bigoni et al. (2000) found analytical solutions for elastic-plastic solids with anisotropic elasticity. Rudnicki (1983) discussed conditions for the onset of strain localization in the case of partly drained behavior. Perić and Rasheed (2007) found analytical solutions for the inception of strain localization in fiber reinforced single phase elastic-plastic materials. Mathematical forms of solutions found by Perić and Rasheed (2007) and by Runesson et al. (1996) are similar in that both, the incompressibility constraint in undrained loading and presence of fibers in one phase materials were found to delay the onset of strain localization. The authors have not been able to identify any existing analytical solutions for inception of strain localization in unsaturated soils.

Borja (2004) developed a mathematical framework for numerical analysis of three-phase deformation and strain localization of partially saturated porous media. The framework was used to detect the onset of strain localization for plane strain compression at the constitutive level. Borja (2004) used the degree of saturation as the effective stress parameter.

Schiava and Etse (2006) conducted a numerical assessment of strain localization in unsaturated soils at constant suction. They used Terzaghi's principle of effective stress for soils subjected to suctions ranging from 0 to $400 \mathrm{kPa}$, thus effectively modeling a drained response of a saturated soil. It is because they did not provide either the soil description or the material 
parameters that it cannot be concluded whether the actual soil they modeled could realistically still be fully saturated in the corresponding suction range. Callari et al. (2010) simulated the response of a perfectly homogeneous initially fully saturated uniformly deforming soil sample subjected to plane strain compression during which the atmospheric pressure was maintained at the top and bottom boundaries only. They detected the inception of strain localization by using the criterion based on the singularity of the underlying drained acoustic tensor. Callari et al. (2010) stated that strain localization was triggered by a heterogeneous effective stress state induced by fluid flow coupling and no material imperfections were introduced. They used an approximation of a rate of average pore pressure, which appears in Lewis-Schefler effective stress. Ehlers et al. (2004) and Ehlers (2011) developed a computational model for capturing strain localization in variably saturated soils. It is because they regularized this otherwise illposed problem by the use of elastic-viscoplastic soil in the presence of two viscous pore fluids that they did not perform a formal diagnostic analysis for the inception of strain localization. Borja et al. (2013) investigated the effect of spatially varying degree on saturation and density on triggering shear band in clay and sand samples subjected to plane strain compression. They used the degree of saturation as the effective stress parameter.

Buscarnera and di Prisco (2011) found that instability of shallow unsaturated slopes was triggered by a mechanism that embodied characteristics of both, shear localization and static liquefaction. They used the degree of saturation as the effective stress parameter.

In this study analytical solutions for the onset of strain localization in a broad class of unsaturated elastic-plastic porous materials are derived. It is shown that the singularity of the socalled total acoustic tensor is a correct indicator for the inception of strain localization. Furthermore, the analytical solutions for three different drainage conditions including undrained, 
constant water content and drained loadings are also derived. In deriving these solutions the definition of the effective stress proposed by Khalili and Khabbaz (1998) and Khalili et al. (2004), which is based on the substantial amount of experimental evidence, was used. The analytical solutions are implemented into the bounding surface plasticity model for unsaturated soils (Khalili et al. 2008). This constitutive model takes into account the simultaneous flow of water and air as well as their complex interaction with the solid skeleton within a consistent elastic-plastic framework. It is used to simulate the strain localization responses of Bourke silt from Bourke region in New South Wales, Australia subjected to conventional triaxial compression (CTC) and plane strain compression (PSC) under different drainage conditions.

\section{PRELIMINARIES}

The porous medium is assumed to be an unsaturated elastic-plastic material experiencing an infinitesimal strain, and obeying a general non-associative flow rule. A nominal time rate is used rather than any objective rate measure due to the assumption of small strains. The indicial notation is used, thus implying summation convention unless stated otherwise.

\section{Mixture Theory}

Unsaturated soils are mixtures of three independent overlapping continua including a solid phase, which is often referred to as a solid skeleton, and two fluids. One of the fluids is wetting (usually water) and the other is non-wetting (usually air). An unsaturated soil can also be viewed as a porous medium, within which each phase is endowed with its own kinematics, mass and momentum (Eringen and Ingram 1965; Bowen 1976). 
Each constituent has a mass $M_{\alpha}$ and volume $V_{\alpha}$, where the subscript $\alpha$ takes values $s, w, a$ denoting solid, water and air phases respectively. Intrinsic quantities are labeled by subscripts, while partial quantities are labeled by superscripts. The quantities denoting the mixture as a whole are devoid of any indices. For example, the mass density of a mixture $\rho$ is given by:

$\rho=\rho^{s}+\rho^{w}+\rho^{a}$

where

$\rho^{s}=n^{s} \rho_{s} \quad$ and $\quad \rho^{w}=n^{w} \rho_{w} \quad$ and $\quad \rho^{a}=n^{a} \rho_{a}$

Individual volume fractions $n^{\xi}$ are related as follows

$n^{s}+n^{w}+n^{a}=1$

In accordance to Loret and Khalili (2000) the volume content of a phase per unit reference volume of a porous medium $\left(v^{\xi}\right)$ and of fluid mass content of each phase per unit reference volume of porous medium $\left(m_{\xi}\right)$ are defined as:

$v^{\xi}=\frac{V_{\xi}}{V_{0}}=n^{\xi} \frac{V}{V_{0}} \quad$ and $\quad m_{\xi}=\frac{M_{\xi}}{V_{0}}=\rho_{\xi} v^{\xi}$

\section{Definition of Effective Stress}

The definition of effective stress given by Bishop (1959) is adopted herein. It is given by:

$\sigma_{i j}^{\prime}=\sigma_{i j}+p_{a} \delta_{i j}-\chi s \delta_{i j} \quad$ and $\quad s=p_{a}-p_{w}$

whereby the equal effective stress rates applied to an unsaturated porous material and the equivalent single phase porous material produce equal elastic strain rates. Symbols $\delta_{\mathrm{ij}}, \sigma_{i j}^{\prime}, \sigma_{i j}, s$, $p_{w}$, and $p_{a}$ denote Kronecker delta, an effective stress tensor, a total stress tensor, suction, pore water pressure and pore air pressure respectively. Compressive components of stress and strain tensors are negative while fluid pressures are positive in compression. The expression for the 
effective stress parameter $\chi$, which was proposed by Khalili and Khabbaz (1998), and is based on a substantial amount of experimental observations, is adopted. It is given by:

$\chi=\left\{\begin{array}{l}1, \frac{s}{s_{a e}} \leq 1 \\ \left(\frac{s}{s_{a e}}\right)^{-0.55}, \text { else }\end{array}\right\}$

where $s_{a e}$ is the suction value at the air entry. A hydraulic hysteresis in the soil-water characteristic curve is neglected herein for the sake of simplicity. It is noted that Eq. (5) reduces to Terzaghi's effective stress principle for $s=s_{a e}$.

The effective stress rate is obtained as a temporal derivative of Eq. (5) and it is expressed

as:

$\dot{\sigma}_{i j}^{\prime}=\dot{\sigma}_{i j}+\dot{p}_{a} \delta_{i j}-\psi \dot{S} \delta_{i j}$

where function $\psi$ is given by:

$\psi=\left\{\begin{array}{l}1, \frac{s}{s_{a e}} \leq 1 \\ 0.45 \chi, \text { else }\end{array}\right\}$

It is also noted that the effective mean effective stress can be expressed as:

$p^{\prime}=p_{n e t}+\chi s \quad$ where $\quad p_{n e t}=p-u_{a}$

\section{Stress-Strain Relationship}

An unsaturated porous material is assumed to undergo a rate independent elastic-plastic deformation. The relationship between the effective stress and strain equivalent to the one given by Loret and Khalili (2000) is adopted herein. It is given by:

$\dot{\sigma}_{i j}^{\prime}=D_{i j k l} \dot{\varepsilon}_{k l}^{s}-k \frac{D_{i j k l}^{e} g_{k l}}{A}\left(\frac{\partial F}{\partial s} \dot{s}\right) \quad$ and $\quad A=H+f_{i j} D_{i j k l}^{e} g_{k l}$ 
where $D^{e} i j k l$ is an elastic stiffness moduli tensor of the underlying drained solid. $F$ and $G$ are yield and plastic potential functions respectively. Their gradients are denoted by $f_{i j}$ and $g_{i j}$ respectively, while $H$ is the actual hardening modulus. Constant $k$ takes values 1 and 0 for plastic and elastic loadings respectively. It is noted that Eq. (10) describes a broad class of incrementally linear three-invariant non-associated elastic-plastic models with isotropic and/or kinematic hardening. Plastic loading occurs if the following conditions are satisfied

$$
F=0 \quad \text { and } \quad f_{i j} D_{i j k l}^{e} \dot{\varepsilon}_{k l}^{s}+\frac{\partial F}{\partial s} \dot{s}>0
$$

It is noted that the second term in Eq. (10) represents the effect of suction induced hardening. The elastic-plastic stiffness moduli tensor $D_{i j k l}$ is given by

$$
D_{i j k l}=D_{i j k l}^{e}-k \frac{D_{i j s t}^{e} g_{s t} f_{m n} D_{m n k l}^{e}}{A}
$$

A solid phase is endowed with its own macroscopic infinitesimal strain rate tensor $\left(\dot{\varepsilon}_{i j}^{s}\right)$,

which is expressed in terms of a macroscopic velocity $\left(\dot{u}_{i}^{s}\right)$ by using the following standard kinematic relationship

$$
\dot{\varepsilon}_{i j}^{s}=\frac{1}{2}\left(\frac{\partial \dot{u}_{i}^{s}}{\partial x_{j}}+\frac{\partial \dot{u}_{j}^{s}}{\partial x_{i}}\right)
$$

\section{ONSET OF STRAIN LOCALIZATION}

At this point the following additional assumptions are adopted: 1) effective stress is defined by Eq. (5), 2) pore fluids are immiscible, 3) the water phase is incompressible, and 4) solid particles are incompressible. The analysis is presently conducted at the constitutive level, thus implying that drainage conditions apply at the constitutive level as well. Pursuing the standard approach it is assumed that discontinuities or jumps in displacement and pore fluid 
pressure rates can occur across a singular surface $C$ having unit normal $N_{i}^{*}$ at the onset of strain localization. It is assumed that a jump in displacement rate is continuous along a singular surface $C$ having unit normal $N_{i}^{*}$ and no assumptions are made regarding the homogeneity and variation of displacement rate gradient in the vicinity of $C$. These assumptions describe a weak discontinuity and lead to the following jump in the solid strain rate across $C$

$\left[\dot{\mathcal{E}}_{i j}^{s}\right]=\frac{1}{2}\left(c^{*}{ }_{i} N^{*}{ }_{j}+c^{*}{ }_{j} N^{*}{ }_{i}\right)=\frac{1}{2} \gamma^{*}\left(M_{i}^{*} N^{*}{ }_{j}+M^{*}{ }_{j} N^{*}{ }_{i}\right)$

and

$c_{i}^{*}=\gamma^{*} M_{i}^{*}$ and $\left\|M_{i}^{*}\right\|=\sqrt{M_{i}^{*} M_{i}^{*}}=1$ and $\gamma^{*} \geq 0$

where $\gamma^{*}$ is arbitrary constant and $c^{*}{ }_{i}$ is the eigenvector corresponding to the relevant eigenproblem, which is discussed in the following sections. Square brackets are used to indicate a discontinuity or jump exclusively. The asterisk superscript will subsequently be changed into "u", "cw", and "d" to denote undrained, constant water content, and drained conditions respectively. The continuity of the total traction rate across the singular surface $C$ arises from the equilibrium requirement, thus resulting in

$\left[\dot{\sigma}_{i j}\right] N_{j}^{*}=0$

For the analysis of plane strain and axisymmetric conditions it is assumed that $f_{i j}$ and $g_{i j}$ possess equal principal directions. In addition, it is assumed that two principal directions are located in the plane of interest and denoted by indices 1 and 2 . The in-plane components are ordered so that for example $f_{1} \geq f_{2}$, while $f_{3}$ simply denotes the out-of plane component. It follows from the arguments presented by Perić et al. (1992) that the plane strain solutions are also valid for axisymmetry under the condition that the indices 1 and 2 denote major and minor 
directions in the radial plane, and the index 3 denotes the circumferential direction. Under very mild constraints the above assumptions also imply that $g_{1} \geq g_{2}$.

\section{Case (i): Undrained Loading}

Rates of change of pore water and pore air volumes per unit reference volume under the assumptions adopted herein, were given by Loret and Khalili (2000) as:

$\dot{v}^{w}=\psi \dot{\varepsilon}_{v}^{s}+a_{w w} \dot{s} \quad$ where $\quad a_{w w}=n \frac{\partial S_{r}}{\partial s} \quad$ and $\quad n=n^{a}+n^{w}$

and

$\dot{v}^{a}=(1-\psi) \dot{\varepsilon}_{v}^{s}+a_{a w} \dot{s} \quad$ where $\quad a_{a w}=-a_{w w}$

The rate of solid volumetric strain is denoted by $\dot{\varepsilon}_{v}^{s}$ while $S_{r}$ is a degree of water saturation, and $n$ is porosity.

For an undrained loading, rates of change of mass contents of water and air per unit reference volume are equal to zero, thus leading to

$\dot{m}_{w}=\frac{\partial}{\partial t}\left(\rho_{w} v^{w}\right)=\rho_{w}\left(\psi \dot{\varepsilon}_{v}^{s}+n \frac{\partial S_{r}}{\partial s} \dot{s}+\frac{\dot{p}_{w}}{\bar{K}_{w}}\left(\frac{V}{V_{0}}\right)\right)=0 \quad$ where $\quad \bar{K}_{w}=\frac{K_{w}}{n^{w}}$

and

$\dot{m}_{a}=\frac{\partial}{\partial t}\left(\rho_{a} v^{a}\right)=\rho_{a}\left((1-\psi) \dot{\varepsilon}_{v}^{s}-n \frac{\partial S_{r}}{\partial s} \dot{s}+\frac{\dot{p}_{a}}{\bar{K}_{a}}\left(\frac{V}{V_{0}}\right)\right)=0 \quad$ where $\quad \bar{K}_{a}=\frac{K_{a}}{n^{a}}$

$K_{w}$ and $K_{a}$ are intrinsic bulk moduli of the water and air phases respectively. The rates of change of mass densities of pore fluids are given by:

$\dot{\rho}_{\alpha}=\rho_{\alpha} \frac{\dot{p}_{\alpha}}{K_{\alpha}} \quad$ where $\alpha=w, a$

Assuming that water phase is incompressible Eq. (19) gives: 


$$
\dot{s}=\bar{K}_{s} \dot{\varepsilon}_{v}^{s} \quad \text { where } \quad \bar{K}_{s}=-\frac{\psi}{n \frac{\partial S_{r}}{\partial s}}
$$

Adding Eqs. (19) and (20) gives:

$$
\dot{p}_{a}=-\bar{K}_{a} \dot{\varepsilon}_{v}^{s}\left(\frac{V_{0}}{V}\right) \cong-\bar{K}_{a} \dot{\varepsilon}_{v}^{s}
$$

The total stress rate is obtained by combining Eqs. (7), (10), (22) and (23) as:

$$
\dot{\sigma}_{i j}=D^{u}{ }_{i j k l} \dot{\varepsilon}_{k l}^{s}
$$

where $D^{u}{ }_{i j k l}$ is a so called undrained tangent stiffness moduli tensor given by:

$$
D_{i j k l}^{u}=D_{i j k l}-\bar{K}_{s} \frac{\partial F / \partial s}{A} D_{i j t}^{e} g_{s t} \delta_{k l}+\bar{K}_{f} \delta_{i j} \delta_{k l}
$$

where

$$
\bar{K}_{f}=\bar{K}_{a}+\psi \bar{K}_{s}
$$

It is noted that undrained tangent stiffness tensor given in Eq. (25) does not exist in case of fully saturated materials containing incompressible water $\left(\bar{K}_{f}=\infty\right)$. However, as shown in subsequent sections the critical hardening modulus and corresponding orientation of the singular surface $C$ can be obtained even for the incompressible pore water by taking the limit of the solution presented herein.

Runesson and Ottosen (1991) showed that elastic/plastic bifurcation, in which one of the bifurcated fields is experiencing elastic loading while the other is experiencing plastic loading, cannot occur before plastic/plastic bifurcation. The extension of their proof, which is presented in the Appendix, shows that elastic/plastic bifurcation can never occur before plastic/plastic bifurcation for unsaturated porous materials as well. Consequently, plastic/plastic bifurcation is 
considered here, thus leading to the condition for the onset of strain localization given in Eq. (27) below.

$Q^{u}{ }_{i k} c^{u}{ }_{k}=0$

Eq. (27) was obtained by combining Eqs. (14), (16), and (24).

Thus, the inception of strain localization coincides with the singularity of the undrained acoustic tensor $Q^{u}{ }_{i k}$ and $c^{u}{ }_{r}$ is the corresponding eigenvector. The former is given by:

$Q^{u}{ }_{i k}=N_{j} D_{i j k l}^{u} N_{l}=Q_{i k}-\bar{K}_{s} \frac{\partial F / \partial s}{A} b_{i} N_{k}+\bar{K}_{f} N_{i} N_{k}$

where vector $b_{i}$ is defined as:

$b_{i}=N_{j} D_{i j s t}^{e} g_{s t}$

It is noted that the final direction of unit normal $N_{i}$ is not known at this point resulting in the superscript being omitted. The undrained acoustic tensor does not exist in case of fully saturated materials containing incompressible water $\left(\bar{K}_{f}=\infty\right)$. The acoustic tensor of the underlying drained solid is given by:

$Q_{i k}=N_{j} D_{i j k l} N_{l}=Q_{i k}^{e}-\frac{b_{i} a_{k}}{A} \quad$ and $\quad Q^{e}{ }_{i k}=N_{j} D_{i j k l}^{e} N_{l}$

where

$a_{k}=f_{m n} D_{m n j k}^{e} N_{j}$

Combining Eqs. (14) and (22) gives:

$c^{u}{ }_{r} N_{r}-\frac{[\dot{s}]}{\bar{K}_{s}}=0$

The solution for the eigenvector $c^{u}{ }_{r}$ is obtained by multiplying Eq. (27) by the inverse of the acoustic tensor $P_{r i}\left(=Q_{r i}^{-1}\right)$ and combining the result with Eq. (32) thus yielding 
$c_{r}^{u}=\left(\frac{\partial F / \partial s}{A} P_{r i} b_{i}-\frac{\bar{K}_{f}}{\bar{K}_{s}} P_{r i} N_{i}\right)[\dot{s}]$

where $P_{r i}$ is given by:

$P_{r i}=P_{r i}^{e}+\frac{P_{r k}^{e} b_{k} a_{l} P_{l i}^{e}}{A-a_{p} P_{p q}^{e} b_{q}} \quad$ and $\quad P_{r i}^{e}=\left(Q_{r i}^{e}\right)^{-1}$

To obtain the solution for the critical hardening modulus Eq. (33) is substituted into Eq. (32)

resulting in

$N_{r}\left(\frac{\partial F / \partial s}{A} P_{r i} b_{i}-\frac{\bar{K}_{f}}{\bar{K}_{s}} P_{r i} N_{i}\right)-\frac{1}{\bar{K}_{s}}=0$

and the hardening modulus can be explicitly expressed from Eq. (35) as:

$H^{u}=-f_{i j} D^{e}{ }_{i j k l} g_{k l}+a_{i} P_{i j}^{e} b_{j}-\psi^{u} \frac{b_{k} P_{k l}^{e} N_{l}}{N_{p} P_{p q}^{e} N_{q}}\left\{a_{i} P_{i j}^{e} N_{j}-\frac{\bar{K}_{s}}{\bar{K}_{f}}\left(\frac{\partial F}{\partial s}\right)\right\}$

where

$\psi^{u}=\frac{\bar{K}_{f} N_{i} P_{i j}^{e} N_{j}}{1+\bar{K}_{f} N_{p} P_{p q}^{e} N_{q}}$

Combining Eqs. (33), (34) and (36) gives the alternative expression for the eigenvector $c^{u}{ }_{r}$ as:

$c^{u}{ }_{i}=P^{e}{ }_{i r} w_{r}^{u}[\dot{s}]$

where

$$
w^{u}{ }_{r}=\frac{\bar{K}_{f}}{\bar{K}_{s}}\left(N_{r}-\frac{1+\bar{K}_{f} N_{i} P_{i j}^{e} N_{j}}{\bar{K}_{f} b_{p} P_{p q}^{e} N_{q}} b_{r}\right) \text { and } a_{i} P^{e}{ }_{i j} N_{j} \neq \frac{\bar{K}_{s}}{\bar{K}_{f}}\left(\frac{\partial F}{\partial s}\right)
$$

The alternative equivalent expression for the eigenvector $c^{u}{ }_{r}$ is given in Appendix in Eq. (A14).

It is noted that the eigenvector $c^{u}{ }_{i}$ reduces to the one given by Runesson et al. (1996) for an undrained loading of fully saturated soil, which in the limit corresponds to $\bar{K}_{f} / \bar{K}_{s}=1$. 
At this point it is assumed that the elastic behavior is isotropic. The expressions for several pertinent tensors of the underlying drained solid are given in the Appendix. They include elastic stiffness moduli tensor $\left(D^{e}{ }_{i j k l}\right)$, elastic acoustic tensor $\left(Q_{i j}^{e}\right)$ and its inverse $\left(P_{i j}^{e}\right)$. In addition, for the isotropic elasticity the expression for $\psi^{u}$ can be obtained by substituting Eq. (A6) into Eq. (37).

Next, expressions in Eq. (36) are evaluated for isotropic elasticity and the following is obtained after the subsequent rearranging:

$$
\frac{H^{u}}{2 G}=a_{1} N_{1}^{2}+a_{2} N_{2}^{2}-\frac{g_{1} N_{1}^{2}+g_{2} N_{2}^{2}}{1-v}\left\{\left(1+(1-2 v) \psi^{u}\right)\left(f_{1} N_{1}^{2}+f_{2} N_{2}^{2}\right)-\psi^{u} \frac{\bar{K}_{s}}{\bar{K}_{f}}(1-v) \frac{\partial F}{\partial s}\right\}-k^{u}
$$

where $a_{1}$ and $a_{2}$ are given by:

$a_{\alpha}=2 f_{\alpha} g_{\alpha}+\frac{v\left(1-\psi^{u}\right)}{1-v}\left(f_{v} g_{\alpha}+f_{\alpha} g_{v}\right)$ and $\alpha=1,2$ (no summation)

and $f_{v}$ and $g_{v}$ are given in Eq. (A5) while $k^{u}$ is given by:

$$
k^{u}=\frac{v(1-2 v)+v^{2} \psi^{u}}{(1-v)(1-2 v)} f_{v} g_{v}+f_{1} g_{1}+f_{2} g_{2}+f_{3} g_{3}-\psi^{u}\left(\frac{\bar{K}_{s}}{\bar{K}_{f}}\right)\left(\frac{\partial F}{\partial s}\right) \frac{v g_{v}}{1-2 v}
$$

Differentiating $H^{u} / 2 G$ given in Eq. (40) with respect to $N_{l}^{2}$ gives:

$$
\frac{d}{d\left(N_{1}^{2}\right)}\left(\frac{H}{2 G}\right)=\frac{1}{1-v}\left\{d_{1}^{u}-\left(d_{1}^{u}-d_{2}^{u}\right) N_{1}^{2}\right\}=0
$$

where scalars $d^{u}{ }_{1}$ and $d^{u}{ }_{2}$ are given by:

$$
d^{u}{ }_{1}=\left(f_{1}-f_{2}\right)\left(g_{1}+v g_{3}\right)+\left(g_{1}-g_{2}\right)\left(f_{1}+v f_{3}\right)-\psi^{u} r^{u}
$$


$d^{u}{ }_{2}=\left(f_{1}-f_{2}\right)\left(g_{2}+v g_{3}\right)+\left(g_{1}-g_{2}\right)\left(f_{2}+v f_{3}\right)-\psi^{u} r^{u}$

and scalars $r_{1}^{u}$ and $r_{2}^{u}$ are given by:

$$
r_{1}^{u}=\left(f_{1}-f_{2}\right)\left\{v g_{v}+(1-2 v) g_{2}\right\}+\left(g_{1}-g_{2}\right)\left\{v f_{v}+(1-2 v) f_{2}-\frac{1-v}{\psi}\left(\frac{\partial F}{\partial s}\right)\left(g_{1}-g_{2}\right)\right\}
$$

$$
r_{2}^{u}=\left(f_{1}-f_{2}\right)\left\{v g_{v}+(1-2 v) g_{1}\right\}+\left(g_{1}-g_{2}\right)\left\{v f_{v}+(1-2 v) f_{1}-\frac{1-v}{\psi}\left(\frac{\partial F}{\partial s}\right)\left(g_{1}-g_{2}\right)\right\}
$$

And subtracting Eq. (45) from Eq. (44) gives:

$$
d^{u}{ }_{1}-d^{u}{ }_{2}=2\left\{1+(1-2 v) \psi^{u}\right\}\left(f_{1}-f_{2}\right)\left(g_{1}-g_{2}\right) \geq 0
$$

By again differentiating Eq. (43) gives:

$\frac{d^{2}}{d\left(n_{1}^{2}\right)^{2}}\left(\frac{H}{2 G}\right)=-\frac{1}{1-v}\left(d_{1}^{u}-d_{2}^{u}\right) \leq 0$

thus confirming that possible solutions of Eq. (40) indeed represent the maximum value of $H$.

Next, only the cases corresponding to $f_{1}>f_{2}$ and $g_{1}>g_{2}$ are considered. Eq. (43) then gives the following solution:

$\left(N_{1}^{u}\right)^{2}=\frac{d^{u}}{d^{u}{ }_{1}-d^{u}{ }_{2}} \quad$ and $\quad\left(N_{2}^{u}\right)^{2}=-\frac{d^{u}{ }_{2}}{d^{u}{ }_{1}-d^{u}{ }_{2}}$

Eq. (50) is valid whenever $0 \leq\left(N_{1}^{u}\right)^{2} \leq 1$ (or $1 \geq\left(N_{2}^{u}\right)^{2} \geq 0$ ), which corresponds to

$d_{1}^{u} \geq 0 \quad$ and $\quad d^{u}{ }_{2} \leq 0$ 
The orientation of a singular surface $C$ representing a boundary of a deformation band is defined by the angle $\theta^{u}$ between its unit normal and $x_{2}$-axis, which is equal to the angle between the singular surface and $x_{1}$-axis. The angle is defined by:

$\tan ^{2} \theta^{u}=\frac{\left(N_{1}^{u}\right)^{2}}{\left(N_{2}{ }_{2}\right)^{2}}=-\frac{d^{u}}{d^{u}{ }_{2}}$

Two extreme cases occur when conditions in Eq. (51) are not satisfied. First, if $d^{u}{ }_{1} \leq 0$ the solution $N^{u}{ }_{1}=0$ and $N^{u}{ }_{2}=1$ corresponding to $\theta^{u}=0^{\circ}$ is obtained. Similarly, when $d^{u}{ }_{2} \geq 0 \quad N^{u}{ }_{1}=$ 1 and $N_{2}^{u}=0$ corresponding to $\theta^{u}=90^{\circ}$ is obtained. In all cases discussed above the corresponding critical hardening modulus is obtained by substituting the relevant values of $N^{u}{ }_{1}$ and $N^{u}{ }_{2}$ into Eq. (40).

It is noted that the expression for the critical hardening modulus of fully saturated porous material given by Runesson at el. (1996) in their Eq. (31) is fully recovered from Eq. (40) herein by setting $\psi^{u}=1\left(\bar{K}^{f}=\infty\right.$ and $\left.\bar{K}_{s} / \bar{K}^{f}=1\right)$ and $\partial F / \partial s=0$ in Eqs. (40) and (42). In addition, the bifurcation directions given by Runesson et al. (1996) in their Eqs. (41) and (43) are also fully recovered from Eqs. (50) and (52) herein by setting $\partial F / \partial s=0$ in Eqs. (46) and (47) and $\psi^{u}=1$ in Eqs. (44) and (45). Furthermore, the expression for the critical hardening modulus of monophasic material given by Runesson et al. (1991) in their Eq. (53) is fully recovered from Eq. (40) herein by setting $\psi^{u}=0$ in Eqs. (40), (41) and (42). Finally, bifurcation directions given by Runesson et al. (1991) in their Eqs. (58) and (59) are also fully recovered from Eqs. (50) and (52) herein by setting $\psi^{u}=0$ in Eqs. (44) and (45) and $\partial F / \partial s=0$ in Eqs. (46) and (47).

\section{Case (ii): Constant Water Content}


For the constant water content loading Eq. (20) is not valid any more, thus rendering Eq. (23) invalid as well. This leads to further simplification of Eq. (26) into

$$
\bar{K}_{f}=\psi \bar{K}_{s}
$$

Eq. (53) is substituted into Eqs. (24), (25), (27), (28) and Eqs. (32) through (52) while simultaneously changing the superscript "u" into "cw" thereby signifying the constant water content loading. Bifurcation directions are given by the unit normal $N^{c w}{ }_{i}$, the expressions for which are provided by Eq. (50) whenever the conditions in Eq. (51) are satisfied. Otherwise, if $d^{c w}{ }_{1} \leq 0$ we obtain the solution $N^{c w}{ }_{1}=0$ and $N^{c w}{ }_{2}=1$ corresponding to $\theta^{c w}=0^{\circ}$. Similarly, when $d^{c w}{ }_{2} \geq 0$ we obtain $N^{c w}{ }_{1}=1$ and $N^{c w}{ }_{2}=0$ corresponding to $\theta^{c w}=90^{\circ}$. In all cases discussed above the corresponding critical hardening modulus is obtained by substituting the relevant values of $N^{c w}{ }_{1}$ and $N^{c w}{ }_{2}$ into Eq. (40).

\section{Case (iii): Drained loading}

A drained test is a constant suction test, thus simplifying Eq. (10) into

$$
\dot{\sigma}_{i j}^{\prime}=D_{i j k l} \dot{\dot{\varepsilon}}_{k l}^{s}
$$

Consequently, the tangent stiffness tensor of the underlying drained solid is the relevant stiffness

tensor for drained loading $\left(D_{i j k l}=D^{d}{ }_{i j k l}\right)$ whereby the rate of the effective stress tensor is equal to the rate of the total stress tensor. It is noted that the effective stress tensor is defined by Eq. (5), thus accounting for the presence of constant suction. Eqs. (19) and (20) are not valid for drained loading, thus rendering Eqs. (22), (23), (26), (32), (33), (35), (38) and (39) invalid as well. Consequently, the terms containing $\bar{K}_{s}, \bar{K}_{f}$ and $\partial F / \partial s$ are now omitted from Eqs. (25), (27), (28), (36) and (40) through (52) whereby $\psi^{u}$ is set to zero. A solution for the eigenvector is provided by Eq. (A15) whereby $\psi^{u}$ is again set to zero in Eq. (A16). While these rearrangements 
are carried the superscript " $u$ " is changed to " $d$ ", thus indicating the applicability to drained loading. Bifurcation directions are given by the unit normal $N^{d}{ }_{i}$, the expressions for which are provided by Eq. (50) whenever the conditions in Eq. (51) are satisfied. Otherwise, if $d^{d}{ }_{1} \leq 0$ the solution $N^{d}{ }_{1}=0$ and $N^{d}{ }_{2}=1$ is obtained corresponding to $\theta^{d}=0^{\circ}$. Similarly, when $d^{d}{ }_{2} \geq 0 \quad N^{d}{ }_{1}=$ 1 and $N_{2}^{d}=0$ is obtained corresponding to $\theta^{d}=90^{\circ}$. In all cases discussed above the corresponding critical hardening modulus is obtained by substituting the relevant values of $N^{d}{ }_{1}$ and $N_{2}^{d}$ into Eq. (40).

\section{APPLICATIONS TO BOURKE SILT}

The above solutions are applied to the bounding surface plasticity model for unsaturated soils developed by Khalili et al. (2008). The essential ingredients of this non-associated plasticity model are: 1) isotropic elasticity, 2) bounding surface separating admissible from inadmissible stress states, 3) loading surface at which the current stress state is located, 4) a plastic potential, which provides the direction of plastic strain, and 5) a hardening rule, which controls the movement of the current stress state towards the image point on the bounding surface as well as locations and sizes of loading and bounding surfaces. The model was calibrated against the experimental results of drying test and a series of suction controlled CTC tests on Bourke silt (Uchaipichat 2005) from Bourke region of New South Wales, Australia. These tests were carried out in a modified Bishop-Wesley triaxial cell capable of independent measurement and control of sample temperature, pore-water pressure, pore-air pressure, sample volume change and water volume change. The samples were prepared by static compaction at a water content dry of 
optimum. An isotropic preconsolidation effective stress of $200 \mathrm{kPa}$ was applied to all samples, followed by unloading to desired initial net stresses and application of target suctions.

An excellent performance of the bounding surface plasticity model is illustrated in Figure 1, which depicts comparisons between the experimental results and model predictions. The later were obtained for the sets of model parameters shown in Tables 1 and 2. $M$ is the slope of critical state line, and $v$ is Poisson ratio. The parameter $A$ is associated with the shape of a plastic potential, while parameters $N$ and $R$ are associated with the shapes of loading and bounding surfaces. The parameter $k_{m}$ appears in the expression for hardening modulus, while the parameter $\lambda_{p}$ is the exponent, which describes the relationship between the effective saturation and suction (Brooks and Corey 1964). The value of suction at the air entry $\left(s_{a e}\right)$ was found to be equal to 18 $\mathrm{kPa}$. A dependence of the intercept $(N)$ and slope $(\lambda)$ of the isotropic consolidation line on suction is shown in Table 2 .

A diagnostic tool for detection of strain localization was developed by implementing the analytical solutions presented herein into the constitutive driver for bounding surface plasticity model. In particular, the solutions for the critical hardening modulus, unit normal $N^{*}$, and the eigenvector angle $\alpha^{*}$ between the unit normal $N^{*}{ }_{i}$ and the eigenvector $c^{*}{ }_{i}$ were found for every load step. To simulate CTC tests both total horizontal stresses were kept constant for the duration of loading, while the vertical compressive strain was increased at the constant rate. The PSC tests differed from the CTC tests in that the strain in one of the horizontal directions was equal to zero for the duration of loading. Neither air nor water phase were allowed to drain in undrained tests, only air was allowed to dry in constant water content tests, while air and water were both allowed to drain in drained tests. The onset of strain localization was signified by the equality between the actual and critical hardening moduli. Figure 2 depicts the minimum differences 
between the critical and actual hardening moduli for a multiplicity of CTC tests including different drainage conditions up to an axial strain of $15 \%$. It can be seen that the smallest minimum difference is slightly above $4000 \mathrm{kPa}$, thus indicating that no single onset of strain localization was detected in CTC tests regardless of drainage conditions. In addition, the experimental data depicted in Figure $1 \mathrm{~b}$ that were used for calibration of the constitutive model show absence of any significant post-peak drop, thus also suggesting that no strain localization occurred in conventional triaxial tests on Bourke silt.

On the contrary the diagnostic analysis detected an inception of strain localization below the axial strain of $15 \%$ in every single drained PSC test with the initial net mean stresses of 30 $\mathrm{kPa}$ and $100 \mathrm{kPa}$ with suctions ranging from $0 \mathrm{kPa}$ to $250 \mathrm{kPa}$. Among all these tests, which are depicted in Figures 3 and 4, the earliest onset was detected at $0.7 \%$ of axial strain (Figure 3), and the latest at 13\% axial strain (Figure 4). Figures 3 and 4 show that increase in suction delays the onset of strain localization. This is most likely primarily due to the effect of suction on the overconsolidation ratio (OCR), which is defined as a ratio of the past maximum and current mean effective stress. The earliest onset corresponds to the initial OCR of 6.7 (Figure 3), thus indicating a heavily overconsolidated soil, while the latest onset corresponds to the OCR of 1.26 (Figure 4), thus indicating a lightly overconsolidated soil.

Figure 5 illustrates the effects of drainage conditions on the inception of strain localization in PSC tests with the initial net mean stress of $100 \mathrm{kPa}$ and the initial suction of 50 $\mathrm{kPa}$. It can be observed that there is no significant difference between the predicted onsets of strain localization in drained test and constant water content test whereby the former occurs at an axial strain of $12.8 \%$ while the later occurs at an axial strain of $12.5 \%$. However, the inception of strain localization in undrained test occurs much earlier, at $6.3 \%$ of axial strain. Thus, in 
unsaturated soils the undrained condition can promote the strain localization by decreasing the initial suction. On the contrary, it was found by Runesson et al. (1996) that undrained condition in saturated soils suppresses the onset of strain localization because it is synonymous with the zero volume change, which imposes a strict constraint on deformation.

Figure 6 illustrates dependence of the critical strain, which is the value of axial strain at onset of strain localization, on the initial net mean stress, initial suction, and drainage conditions. Two types of behaviors are observed: 1) the range of the initial net mean stress in which the critical strain is approximately constant, and 2) the range of initial net mean stresses in which the critical strain increases in a non-linear fashion with the increasing initial net mean stress. For example, the former range corresponds to critical strains smaller than $2 \%$, which are possible only for the initial suctions of $50 \mathrm{kPa}$ and $150 \mathrm{kPa}$ for drained and constant water content tests. For undrained tests the critical strain is smaller than $2 \%$ for the initial suctions of $50 \mathrm{kPa}, 150$ $\mathrm{kPa}$ and $300 \mathrm{kPa}$. Furthermore, this early onset persists up to the initial mean net stress of about $50 \mathrm{kPa}$ in undrained tests, thus corresponding to the minimum initial OCR of 2.55. In drained and constant water tests the early onset persists up to the initial mean net stress of about $40 \mathrm{kPa}$, thus corresponding to the minimum initial OCR of 2.92. This again illustrates larger susceptibility of undrained tests to strain localization.

Figure 7 presents orientations of deformation bands, whereby the critical angle depicted on y-axis is the angle between a deformation band and horizontal (or major principal stress) direction. In drained and constant water content tests, the critical angle sharply decreases from the maximum of $46.7^{\circ}$ and $46.6^{\circ}$ respectively with an increase of the initial net mean stress. It remains constant at $44.7^{\circ}$ at higher initial net mean stresses. In undrained tests the critical angle initially also decreases sharply from the maximum value of $46.6^{\circ}$. It subsequently starts to 
asymptotically approach the value of $44.5^{\circ}$ with an increase of the initial net mean stress. Finally, Figure 8 depicts the angle $\alpha^{*}$ between the unit normal to the deformation band $N^{*}{ }_{i}$ and the eigenvector $c^{*}{ }_{i}$, which indicates the mode or type of deformation band. The angle is obtained from the definition of a scalar product as follows:

$\alpha^{*}=\cos ^{-1}\left(M_{i}^{*} N^{*}{ }_{i}\right)$

According to Eq. (14) a jump in the volumetric strain rate across the singular surface $C$ is given by

$\left[\dot{\varepsilon}_{v}^{s}\right]=\gamma^{*} M_{i}^{*} N^{*}{ }_{i}=\gamma^{*} \cos \alpha^{*}$

Thus, a positive jump in the volumetric strain rate indicates a dilatant deformation band, a negative jump signifies a contractant deformation band while a zero jump indicates a pure shear band. According to Figure 8 slightly dilatant shear bands occur only for the initial suction of 50 $\mathrm{kPa}$ at the initial net mean stresses smaller than about $31.5 \mathrm{kPa}$, thus corresponding to the initial overconsolidation ratios larger than 3.33 and indicating heavily overconsolidated soils. The inception of strain localization occurs in the form of pure shear band for the initial suction of 50 $\mathrm{kPa}$ and the initial net mean stresses of $28.6 \mathrm{kPa}, 25.7 \mathrm{kPa}$ and $31.5 \mathrm{kPa}$ in drained, constant water content and undrained tests respectively. For all other initial conditions considered the onset of strain localization is characterized by slightly contractant shear bands.

\section{CONCLUSIONS}

Analytical solutions for the onset of strain localization in unsaturated soils subjected to different drainage conditions have been derived for a broad class of elastic-plastic material models. It is shown the solution for an undrained loading has the most complex form and that previously derived solutions for undrained loadings of fully saturated and for monophasic 
materials can be fully recovered from the solutions derived herein simply by appropriately adjusting bulk moduli of pore fluids. A diagnostic tool for detection of strain localization was developed by implementing the above solutions into the constitutive driver for bounding surface plasticity model developed by Khalili et al. (2008). The tool was used to further illustrate the strain localization behavior of Bourke silt, thus leading to the following conclusions:

1) No strain localization was detected in CTC tests regardless of drainage condition, thus deeming CTC loading resistant to strain localization.

2) On the contrary, the inception of strain localization was detected in every single PSC test. Among drained, constant water content and undrained tests starting from equal initial conditions the earliest onset of strain localization was found in undrained tests. This is contrary to the strain localization behavior observed in fully saturated soils (Runesson et al., 1996) whereby undrained condition was found to delay the onset of strain localization.

3) Two types of dependence of the critical strain on the initial net mean stress have been identified. First, there is a range of the initial net mean stress in which the critical strain is approximately constant and very low, thus indicating the early onset. This range coincides with the initially heavily overconsolidated samples. Second, there is a range of the initial net mean stresses in which the critical strain increases in a non-linear fashion with an increasing initial net mean stress. This range corresponds to the initially lightly to normally overconsolidated samples. 4) At the onset of strain localization deformation bands were found to be slightly contractant shear bands for the most part. They formed angles larger than $43.5^{\circ}$ with the major principal stress direction, whereby those detected in drained tests were only slightly steeper than those found in constant water content tests, which in turn were steeper than those detected in undrained tests. Dilatant shear bands were detected only for the smallest initial suction considered ( $50 \mathrm{kPa})$ 
in the range of the initial net mean stresses smaller than $31.5 \mathrm{kPa}$, which essentially corresponds to the highly overconsolidated samples with a minimum OCR of 3. 7. They made angles smaller than $46.7^{\circ}$ with the major principal stress direction. Pure shear bands were detected only in tests with initial suction of $50 \mathrm{kPa}$ for all drainage conditions considered herein.

\section{ACKNOWLEDGEMENT}

The first author gratefully acknowledges the financial support and hospitality provided by University of New South Wales, Sydney, Australia. The second author gratefully acknowledges the support received from Australian Research Council (Grant No. DE 130100457).

\section{REFERENCES}

Benallal, A., Comi, C., (2002). "Perturbation growth and localization in fluid-saturated inelastic porous media under quasi-static loadings." J. of the Mech. and Phys. of Solids, 51, 851-899. Bésuelle, P. (2001). "Compacting and dilating shear bands in porous rock: Theoretical and experimental conditions." J. of Geophysical Research, 106(B7), 13,435-13,442.

Bigoni, D., and Hueckel, T. (1991a). "Uniqueness and Localization-I. Coupled Elastoplasticity." Int. J. Solids and Structures, 28(2), 215-224.

Bigoni, D., and Hueckel, T. (1991b). "Uniqueness and Localization-II. Associative and NonAssociative Elastoplasticity.” Int. J. Solids and Structures, 28(2), 197-213.

Bigoni, D., Loret, B., and Radi, E. (2000). "Localization of deformation in plane elastic-plastic solids with anisotropic elasticity." J. of the Mech. and Phys. of Solids, 48, 1441-1446. Bishop, A.W. (1959). “The principle of effective stress.” Tecknish Ukeblad, 106, 859-863. 
Borja, R.I. (2004). "Cam-Clay plasticity. Part V: A mathematical framework for three-phase deformation and strain localization analyses of partially saturated porous media." Comp. Methods Appl. Mech. and Engrg. , 193, 5301-5338.

Borja, R.I., Song, X., and Wu, W. (2013). "Critical state plasticity. Part VII: Triggering a shear band in variably saturated porous media." Comp. Methods Appl. Mech. and Engrg., 261-262, 6682.

Brooks, R. and Corey, A. (1964). "Hydraulic properties of porous media." Hydrology Paper no. 3, Colorado State University, Forth Collins, Colorado.

Bowen, R.M. (1976). “Theory of mixtures.” Continuum Physics, vol. III, A.C. Eringen, ed. Academic Press, New York, 1-127.

Buscarnera, G. and di Prisco, C. (2011). "Stability criteria for unsaturated shallow slopes." Géotechnique Letters 1, 85-90.

Callari, C., Armero, F., and Abati, A., (2010). "Strong discontinuities in partially saturated poroplastic solids." Comput. Methods Appl. Mech. Engrg., 199, 1513-1555.

Ehlers, W., Graf, T., and Ammann, M. (2004). "Deformation and localization analysis of partially saturated soil." Comput. Methods Appl. Mech. Engrg. 193, 2885-2910.

Ehlers, W., Avci, O., and Markert, B. (2011). "Computation of Slope Movements Initiated by Rain Induced Shear Bands in Small Scale Tests and in Situ." Vadose Zone Journal, 10(2), 512525.

Eringen, A.C., Ingram, J.D. (1965). ”A continuum theory for chemically reacting media-I. ”, Int. J. of Engineering Science, 3, 197-212.

Khalili, N. and Khabbaz, M.H. (1998). "A unique relationship for the determination of the shear strength of unsaturated soils." Géotechnique, 48(5), 681-687. 
Khalili, N., Geiser, F., and Blight, G.E. (2004). "Effective stress in unsaturated soils: review with new evidence.” Int. J. Geomechanics, 4(2), 115-126.

Loret, B. and Khalili, N. (2000). “A three-phase model for unsaturated soils.” Int. J. Numer. Anal. Meth. Geomech., 24, 893-927.

Khalili, N., Habte, M.A., and Zagarbashi, S. (2008). “A fully coupled flow deformation model for cyclic analysis of unsaturated soils including hydraulic and mechanical hysteresis." Computers and Geotechnics, 35, 872-889.

Ottosen, N.S., and Runesson, K. (1991). "Properties of Properties of Discontinuous Bifurcation Solutions in Elasto-Plasticity.” Int. J. Solids and Structures, 27(4), 401-421.

Ottosen, N.S., Runesson, K., and Perić, D. (1991). "Discontinuous Bifurcations of Elastic-Plastic Solutions at Plane Stress and Plane Strain.” Int. J. Plasticity, 7, 99-121.

Perić, D., Runesson, K., and Sture. S. (1992). "Evaluation of Plastic Bifurcation for Plane Strain versus Axisymmetry." J. of Engineering Mechanics, ASCE, 118(3), 512-524.

Perić, D., Runesson, K., and Sture. S. (1993). "Prediction of Plastic Localization Using MRSLade Model.” J. of Geotechnical Eng., ASCE, 119, No. 4, 639-661.

Perić, D. and Rasheed, H. (2007). "Localized failure of fibre-reinforced elastic-plastic materials subjected to plane strain loading." Int. J. Numer. Anal. Meth. Geomech, 31(7), 893-916.

Rudnicki, J.W., and Rice, J.R. (1975). “Conditions for Localization of Deformation in Pressuresensitive Dilatant Materials.” J. Mech. Phys. Solids, 23(6), 371-394.

Rudnicki J.W. (1983). “A formulation for studying coupled deformation pore fluid diffusion effects on localization of deformation." Geomechanics, AMD-57, S. Nemat-Nasser ed. ASME, $35-44$.

Runesson, K., Perić, D., and Sture, S. (1996). "Effect of Pore Fluid Compressibility on 
Localization in Elastic-Plastic Porous Solids under Undrained Conditions.” Int. J. Solids

Structures, 33(10), 1501-1518.

Schiava, R., and Etse, G. (2006). "Constitutive Modeling and Discontinuous Bifurcation Assessment in Unsaturated Soils." Journal of Applied Mechanics, 73, 1039-1044.

Uchaipichat, A. (2005). "Experimental investigation and constitutive modeling of thermo-hydromechanical coupling in unsaturated soils". Ph.D. thesis. The University of New South Wales, Sydney, Australia.

Vardoulakis, I. (1996a). "Deformation of water-saturated sand: I. uniform deformation and shear Banding." Géotechnique, 46(3), 441-456.

Vardoulakis, I. (1996b). "Deformation of water-saturated sand: II. Effect of pore water flow and shear banding." Géotechnique, 46(3), 457-472.

\section{Appendix}

Several tensors pertinent to isotropic elasticity are given here. An elastic stiffness moduli tensor $D_{i j k l}^{e}$ is given by:

$D_{i j k l}^{e}=2 G\left\{\frac{1}{2}\left(\delta_{i k} \delta_{j l}+\delta_{i l} \delta_{j k}\right)+\frac{v}{1-2 v} \delta_{i j} \delta_{k l}\right\}$

An elastic acoustic tensor is obtained as:

$Q^{e}{ }_{i j}=N_{k} D^{e}{ }_{i j k l} N_{l}=G\left(\frac{1}{1-2 v} N_{i} N_{j}+\delta_{i j}\right)$

and its inverse $P_{i j}^{e}$ is then given as:

$$
P_{i j}^{e}=\frac{1}{G}\left(-\frac{1}{2(1-v)} N_{i} N_{j}+\delta_{i j}\right)
$$

By substituting Eq. (A1) into Eqs. (28) and (30) the following expressions are obtained 
$a_{i}=2 G\left(f_{i j} n_{j}+\frac{v}{1-2 v} f_{v} n_{i}\right) \quad$ and $\quad b_{i}=2 G\left(g_{i j} n_{j}+\frac{v}{1-2 v} g_{v} n_{i}\right)$

where $f_{v}$ and $g_{v}$ are volumetric portions of the gradients $f_{i j}$ and $g_{i j}$ respectively given by:

$f_{v}=f_{i j} \delta_{i j} \quad$ and $\quad g_{v}=g_{i j} \delta_{i j}$

Finally, by using Eq. (A3) the following is obtained

$N_{i} P^{e}{ }_{i j} N_{j}=\frac{1-2 v}{2 G(1-4)}$

Next, it is shown that elastic/plastic bifurcation cannot occur before plastic/plastic bifurcation for unsaturated porous materials. The undrained loading is addressed first by considering the following eigenvalue problem:

$Q^{u}{ }_{i l} y^{u(i)}{ }_{l}=\lambda^{u(i)} Q^{e u}{ }_{i l} y^{u(i)}{ }_{l} \quad$ where $\quad i=1,2$

$Q^{e u}{ }_{i j}$ the acoustic tensor associated with undrained elastic behavior, i.e.

$Q^{e u}{ }_{i l}=N_{j}^{u} D_{i j k l}^{e u} N_{k}^{u} \quad$ where $\quad Q^{e u}{ }_{i l}=Q_{i l}^{e}+\bar{K}_{f} N^{u}{ }_{i} N^{u}{ }_{l}$

The positive definiteness of elastic stiffness moduli tensor implies the positive definiteness of $Q^{e u}{ }_{i j}$ as follows:

$c^{u}{ }_{i} Q^{e u}{ }_{i l} c^{u}{ }_{l}=c^{u}{ }_{i} N^{u}{ }_{j}\left(D^{e}{ }_{i j k l}+\bar{K}_{f} \delta_{i j} \delta_{k l}\right) N^{u}{ }_{k} c^{u}{ }_{l}=\left[\dot{\varepsilon}_{i j}\right] D^{e}{ }_{i j k l}\left[\dot{\varepsilon}_{k l}\right]+\bar{K}_{f}\left[\dot{\varepsilon}_{k k}\right]^{2}>0$

where $c^{u}{ }_{i}$ is a non-zero arbitrary vector. $Q^{e u}{ }_{i j}$ possesses the symmetric positive definite inverse $P^{e u}$, which is given by:

$P_{i l}^{e u}=P_{i l}^{e}-\frac{\psi^{u}}{N_{p}^{u} P_{p q}^{e} N_{p}^{u}} P_{i j}^{e} N_{j}^{u} N_{r}^{u} P_{r l}^{e}$

Next, Eq. (A7) is multiplied by $P_{r i}^{e u}$, thus yielding the following:

$B_{r l}^{u} y_{l}^{u(i)}=\lambda^{u(i)} y_{r}^{u(i)}$ 
where $B^{u}{ }_{r l}$ is given by:

$$
B_{r l}^{u}=\delta_{r l}-\frac{1}{A}\left(P_{r i}^{e u} b_{i} a_{l}+\bar{K}_{s} \frac{\partial F}{\partial s} P_{r i}^{e u} N_{i}^{u} b_{l}\right)
$$

Eigenvalues can be expressed from Eq. (A11) as:

$$
B^{u}{ }_{r r}=\lambda^{u(1)}+\lambda^{u(2)}=1+\lambda^{u(2)}
$$

Eq. (A7) implies that the first eigenvalue corresponding to elastic response is equal to one, which together with Eq. (A13) gives:

$$
\lambda^{u(2)}=1-\frac{1}{A}\left(a_{r} P_{r i}^{e u} b_{i}+\bar{K}_{s} \frac{\partial F}{\partial s} b_{r} P_{r i}^{e u} N_{i}^{u}\right)
$$

Combining Eqs. (A11), (A12) and (A14) gives:

$$
y_{j}^{u(2)}=\zeta P_{j i}^{e u} b_{i}
$$

where is $\zeta$ arbitrary constant. It is noted that Eq. (A14) provides the alternative expression for eigenvector, which unlike Eq. (33) does not depend on the jump in suction rate. The alternative expression for the critical hardening modulus can be obtained by setting $\lambda^{u(2)}$ from Eq. (A14) equal to zero, which gives the following:

$$
H^{u}=-f_{i j} D_{i j k l}^{e} g_{k l}+a_{i} P^{e u}{ }_{i j} b_{j}-\bar{K}_{s} N_{i} P^{e u}{ }_{i j} b_{j}
$$

Next, it is shown that elastic/plastic (E/P) discontinuous bifurcation can never precede plastic/plastic (P/P) discontinuous bifurcation. Eq. (16) implies that for E/P discontinuous bifurcation in undrained loading the following holds:

$$
N_{j} D^{u}{ }_{i j l} \dot{\varepsilon}_{k l}^{s+}=N_{j} D^{u e}{ }_{i j k l} \dot{\varepsilon}_{k l}^{s-}
$$

where $\dot{\varepsilon}_{k l}^{s+}$ denotes solid strain tensor rate on one side of singular surface $C$, while $\dot{\varepsilon}_{k l}^{s-}$ is the solid strain tensor rate on the other (elastic) side of C. Substituting Eqs. (14), (22), (25) and (29), 
and subsequent rearranging Eq. (A17) the following is obtained:

$Q^{u}{ }_{i k} c^{u}{ }_{k}=\frac{1}{A} \alpha^{\prime} b_{i} \quad$ where $\quad \alpha^{\prime}=f_{p q} D_{p q r s}^{e} \dot{\varepsilon}_{r s}^{s-}+\frac{\partial F}{\partial s} \dot{s}^{-} \leq 0$

The strain tensor rate on the plastic side of $C$ can be expressed as:

$\dot{\varepsilon}_{r s}^{s-}=\dot{\varepsilon}_{r s}^{s+}-\frac{1}{2}\left(c^{u}{ }_{r} N^{u}{ }_{s}+c^{u}{ }_{s} N^{u}{ }_{r}\right) \quad$ and $\quad \dot{s}^{+}=\dot{s}^{-}+[\dot{s}]$

and substituting Eq. (A19) into Eq. (A18) and rearranging gives the following:

$Q^{e u}{ }_{i k} c^{u}{ }_{k}=\frac{1}{A} \beta^{\prime} b_{i} \quad$ where $\quad \beta^{\prime}=f_{p q} D_{p q r s}^{e} \dot{\varepsilon}_{r s}^{s+}+\frac{\partial F}{\partial s} \dot{s}^{+}>0$

with eigenvector $c^{u}{ }_{k}$ given by:

$c^{u}{ }_{k}=\frac{\beta^{\prime}}{A} P^{e u}{ }_{k l} b_{l}$

It is noted that $c^{u}{ }_{k}$ is the same eigenvector as the one given in Eq. (A15) corresponding to $\mathrm{P} / \mathrm{P}$ bifurcation. The only difference is the scaling factor. Now, vector $b_{i}$ is expressed from Eqs. (A18) and (A20). The two expressions are subsequently equalized to give:

$Q_{i k}^{u} c_{k}^{u}=\frac{\alpha^{\prime}}{\beta^{\prime}} Q^{u e}{ }_{i k} c^{u}$

which represents the eigenvalue problem completely identical to that given in Eq. (A7). Clearly, the elastic solution $\left(\lambda^{u(I)}=1\right)$ is not relevant for Eq. (21), which leaves

$$
\lambda^{u(2)}=\frac{\alpha^{\prime}}{\beta^{\prime}} \leq 0
$$

The result given in Eq. (22) implies that elastic/plastic bifurcation can never occur before plastic/plastic bifurcation. 
Table 1. Suction independent parameters of bounding surface plasticity model

\begin{tabular}{|l|l|}
\hline Parameter & Value \\
\hline $\mathrm{M}$ & 1.17 \\
\hline$v$ & 0.25 \\
\hline$A$ & 2.0 \\
\hline$N$ & 3.0 \\
\hline$R$ & 2.0 \\
\hline$k_{m}$ & 200. \\
\hline$\lambda_{p}$ & 0.41 \\
\hline$S_{a e}$ & 18 \\
\hline
\end{tabular}


Table 2. Suction dependent parameters of bounding surface plasticity model

\begin{tabular}{|l|l|l|}
\hline suction, $s(\mathrm{kPa})$ & $\lambda(s)$ & $N(s)$ \\
\hline$s \leq s_{a e}$ & 0.090 & 2.049 \\
\hline 100 & 0.090 & 2.058 \\
\hline 300 & 0.090 & 2.068 \\
\hline
\end{tabular}


Figure 1. Model calibration for Bourke silt: a) Drying path tests

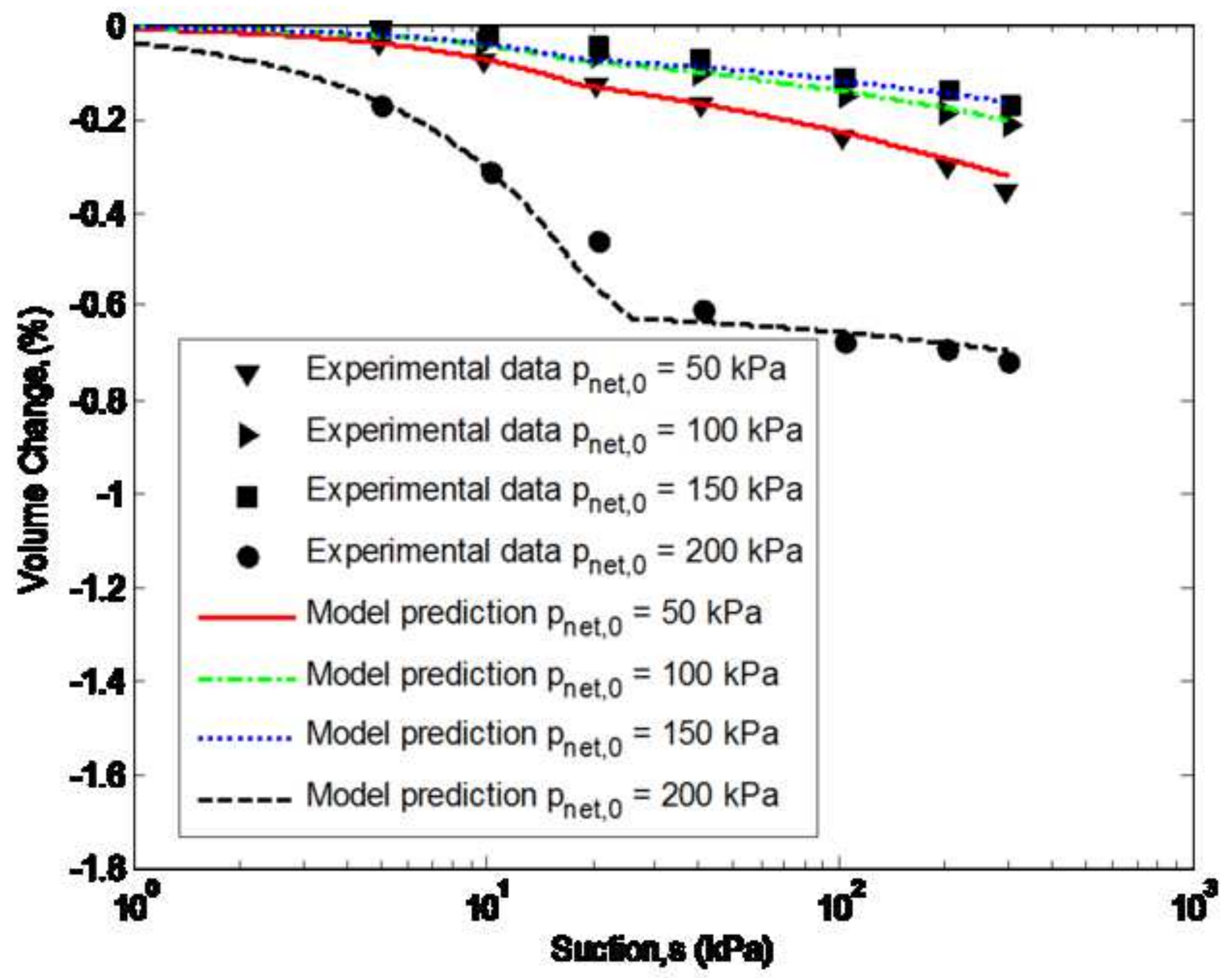




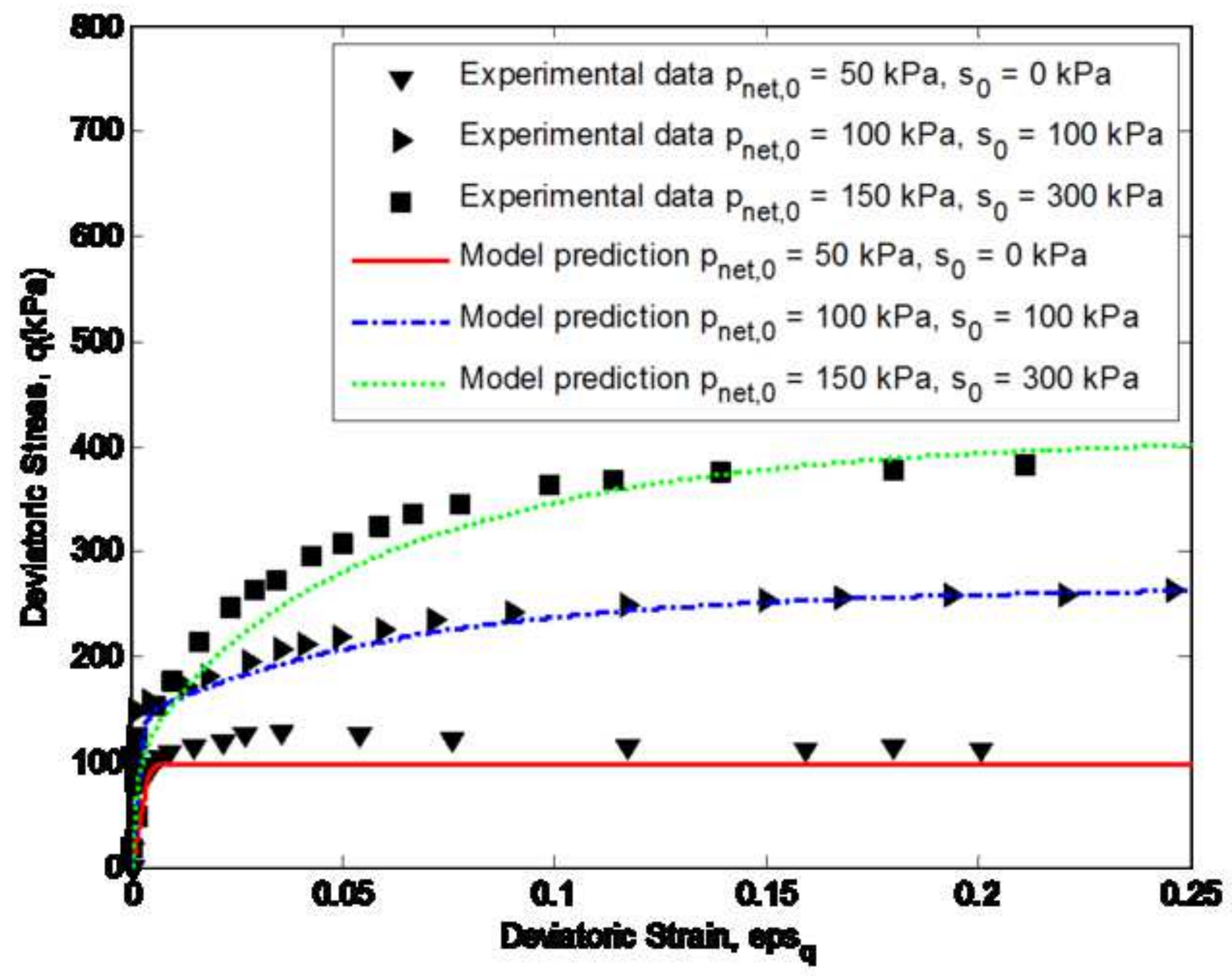


Figure 1. Model calibration for Bourke silt: c) Drained CTC tests (strain-strain response)

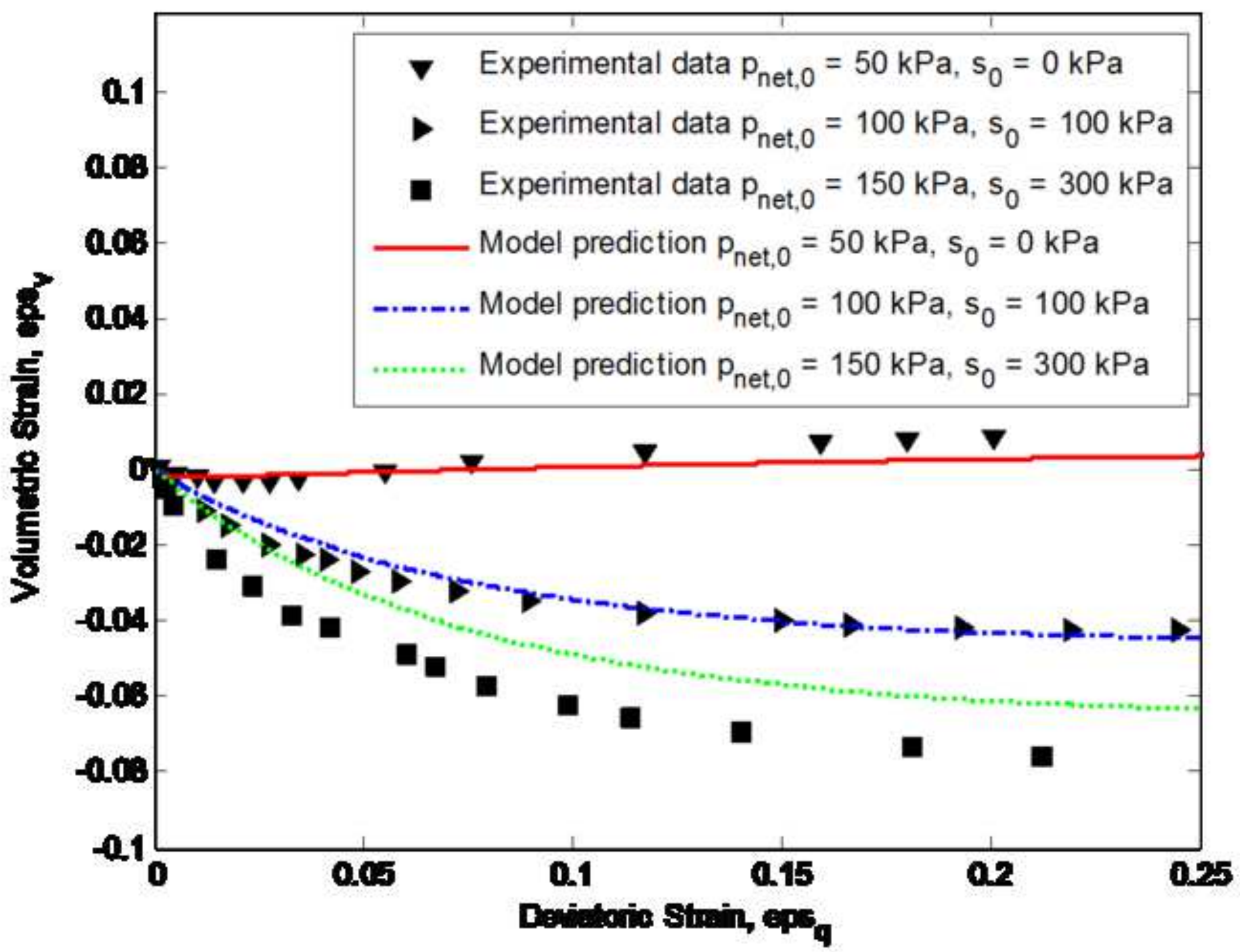


Figure 2. Minimum difference between actual and critical hardening moduli for CTC tests: a) drained

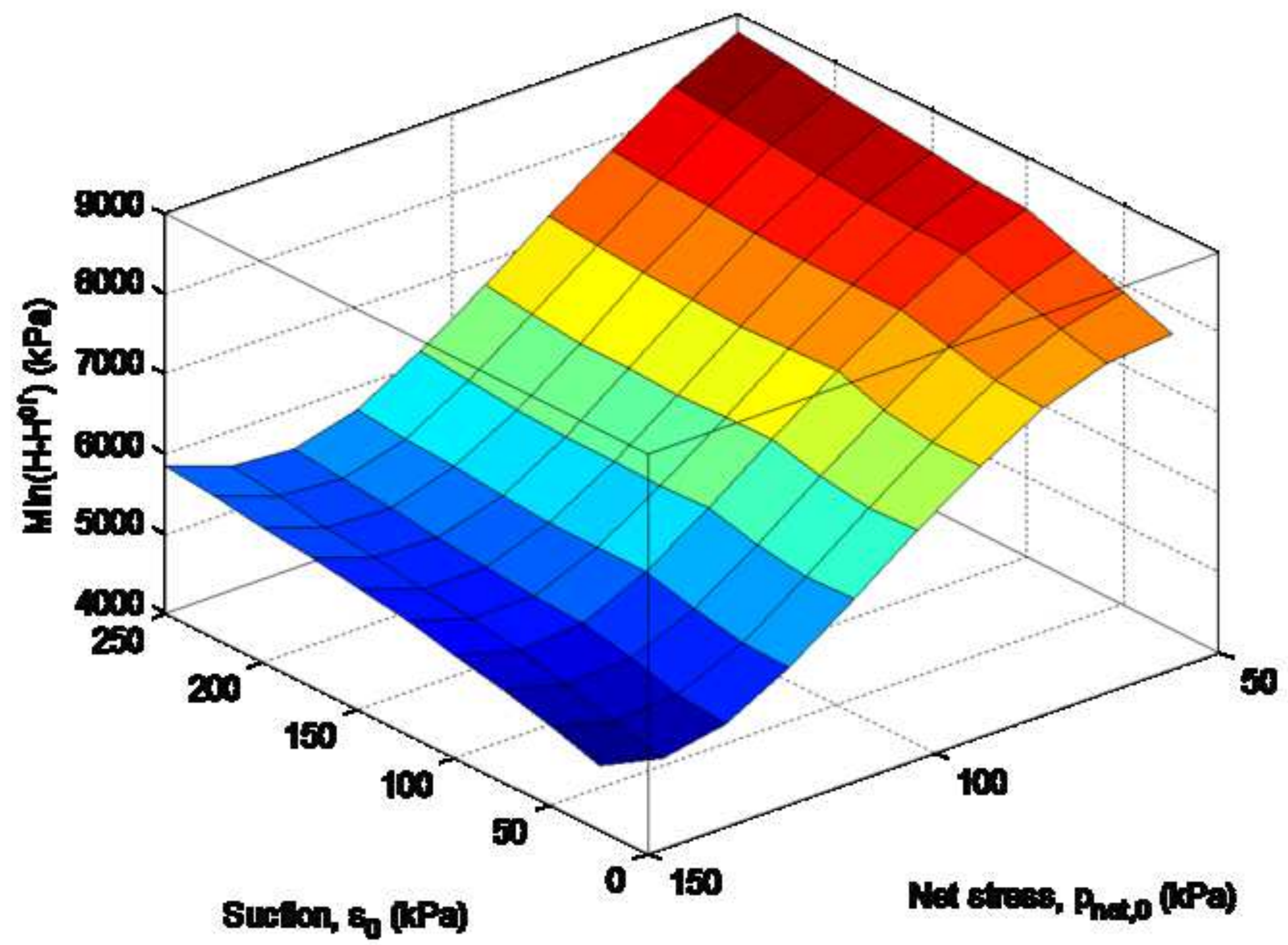


Figure 2. Minimum difference between actual and critical hardening moduli for CTC tests: b) constant water content

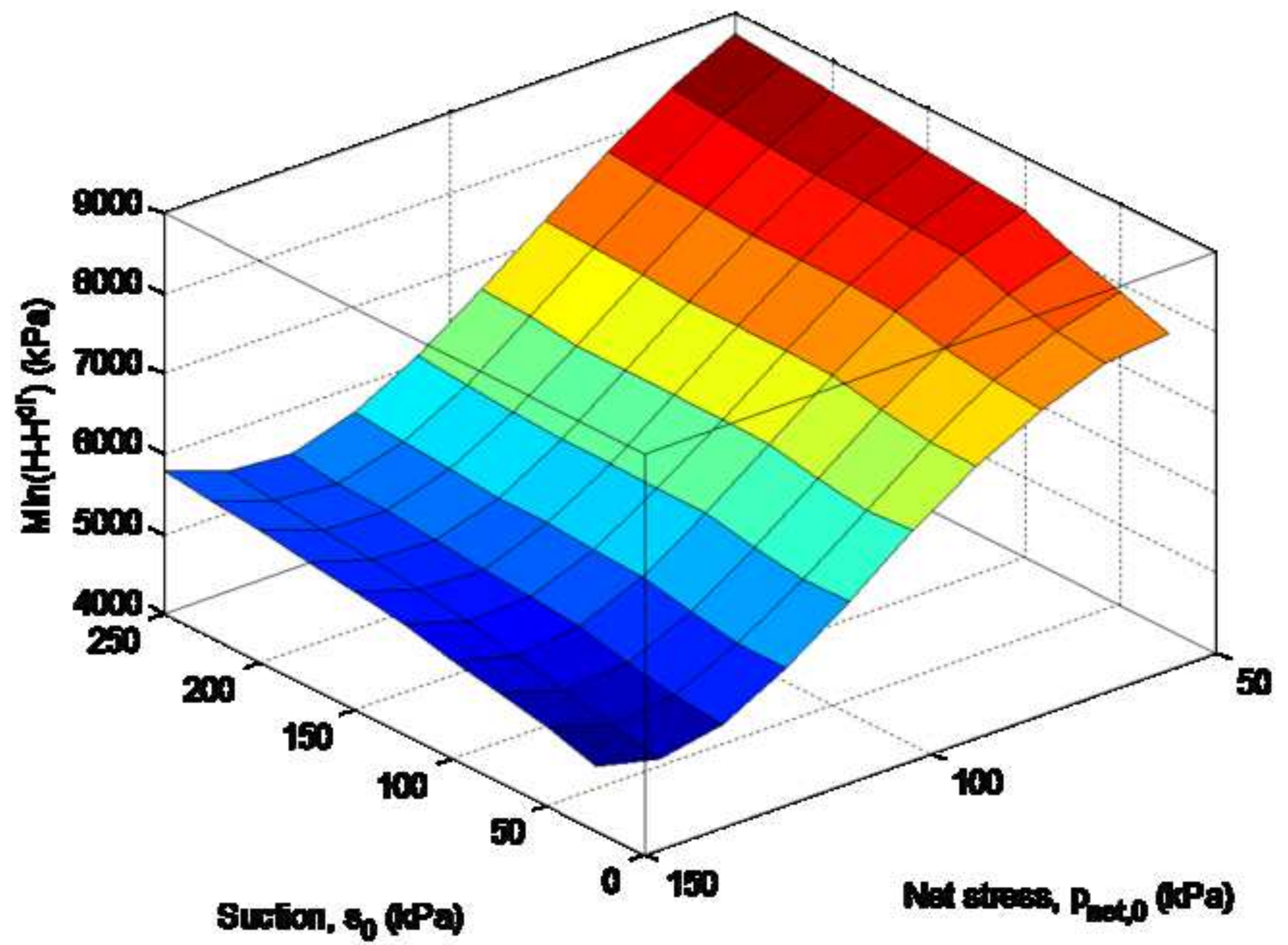


Figure 2. Minimum difference between actual and critical hardening moduli for CTC tests: c) undrained

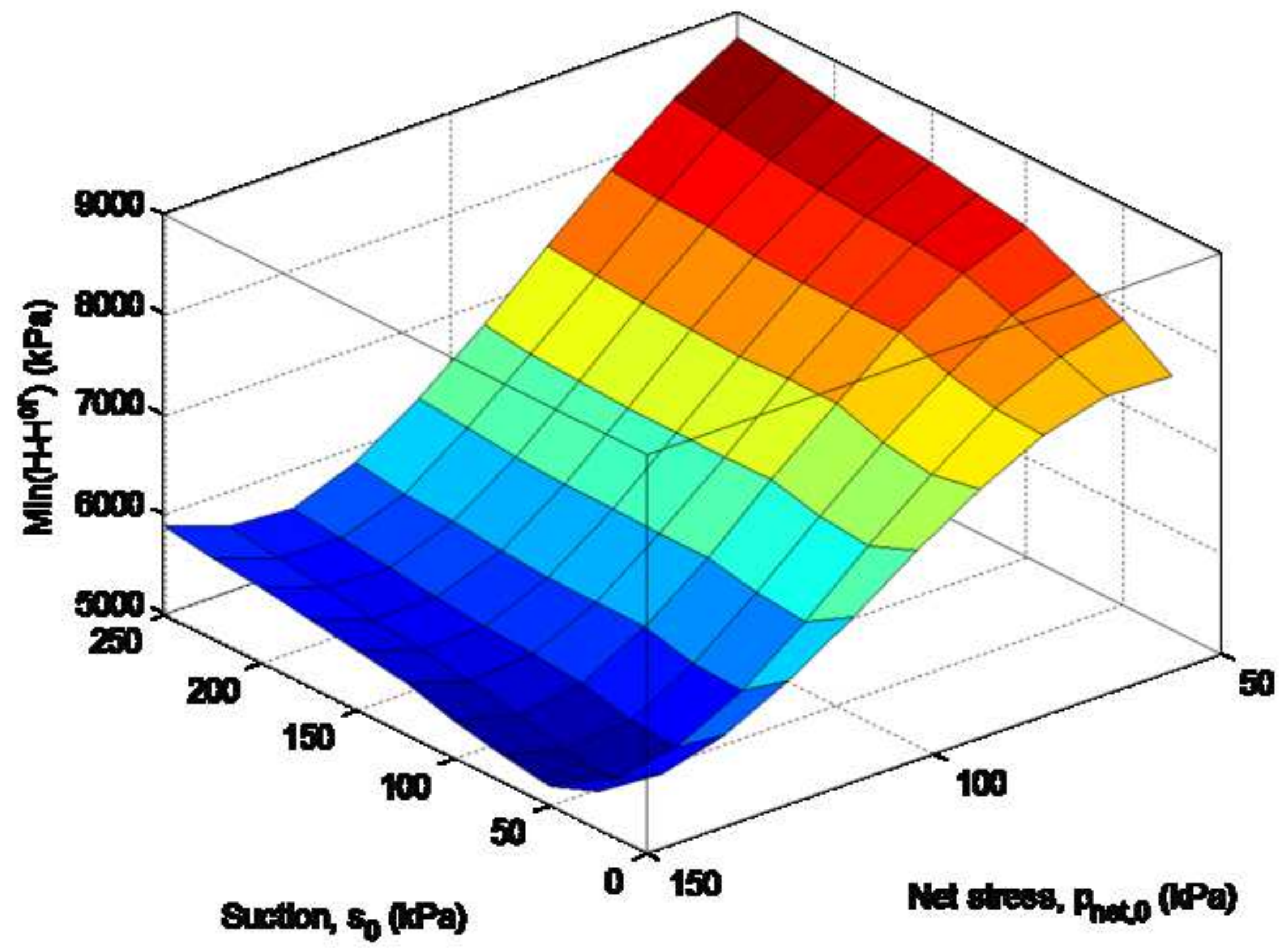


Figure 3. Prediction of the onset of strain localization for drained PSC tests at $p_{n e t, 0}=30 \mathrm{kPa}$

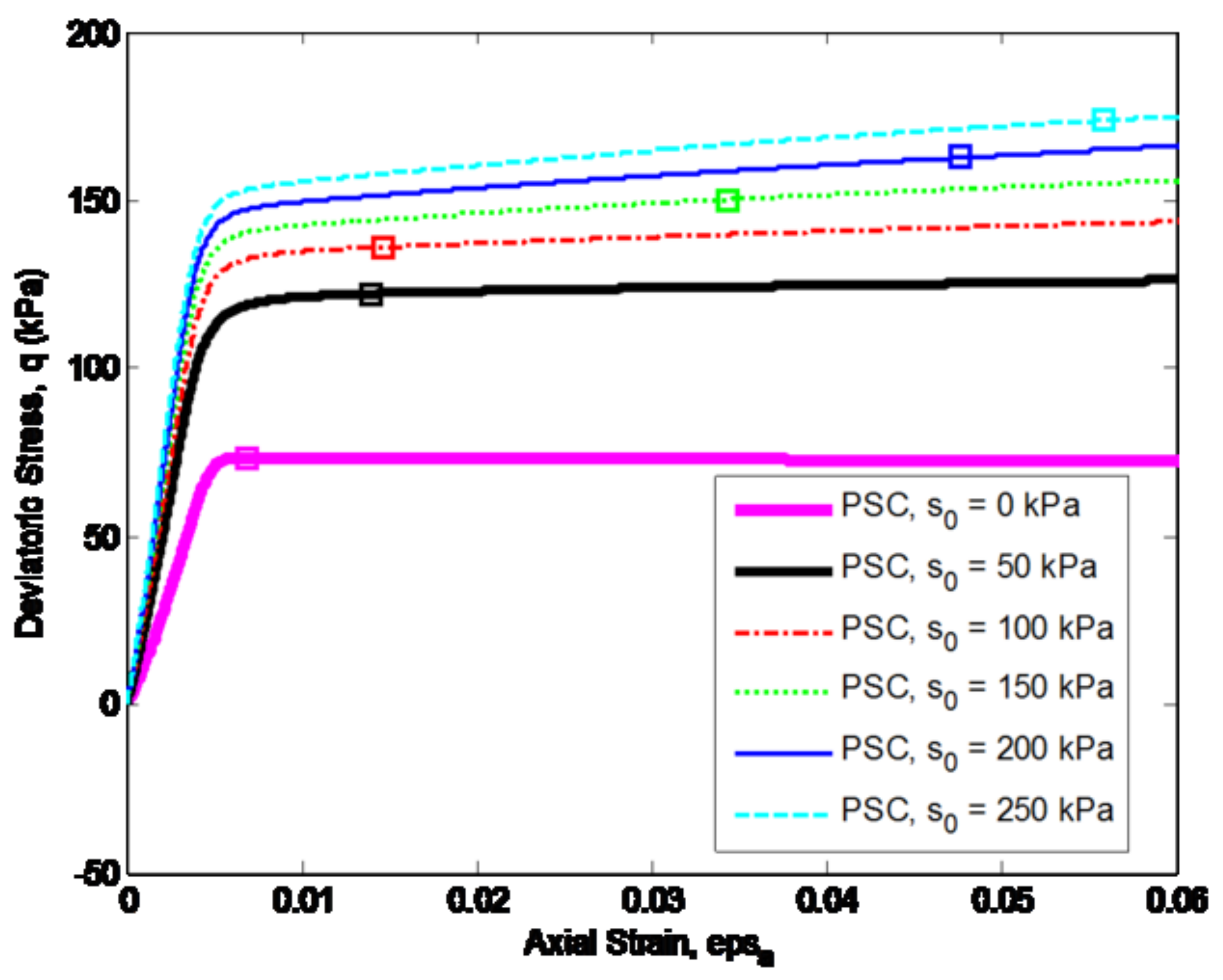


Figure 4. Prediction of the onset of strain localization for drained PSC tests at pnet, $0=100 \mathrm{kPa}$

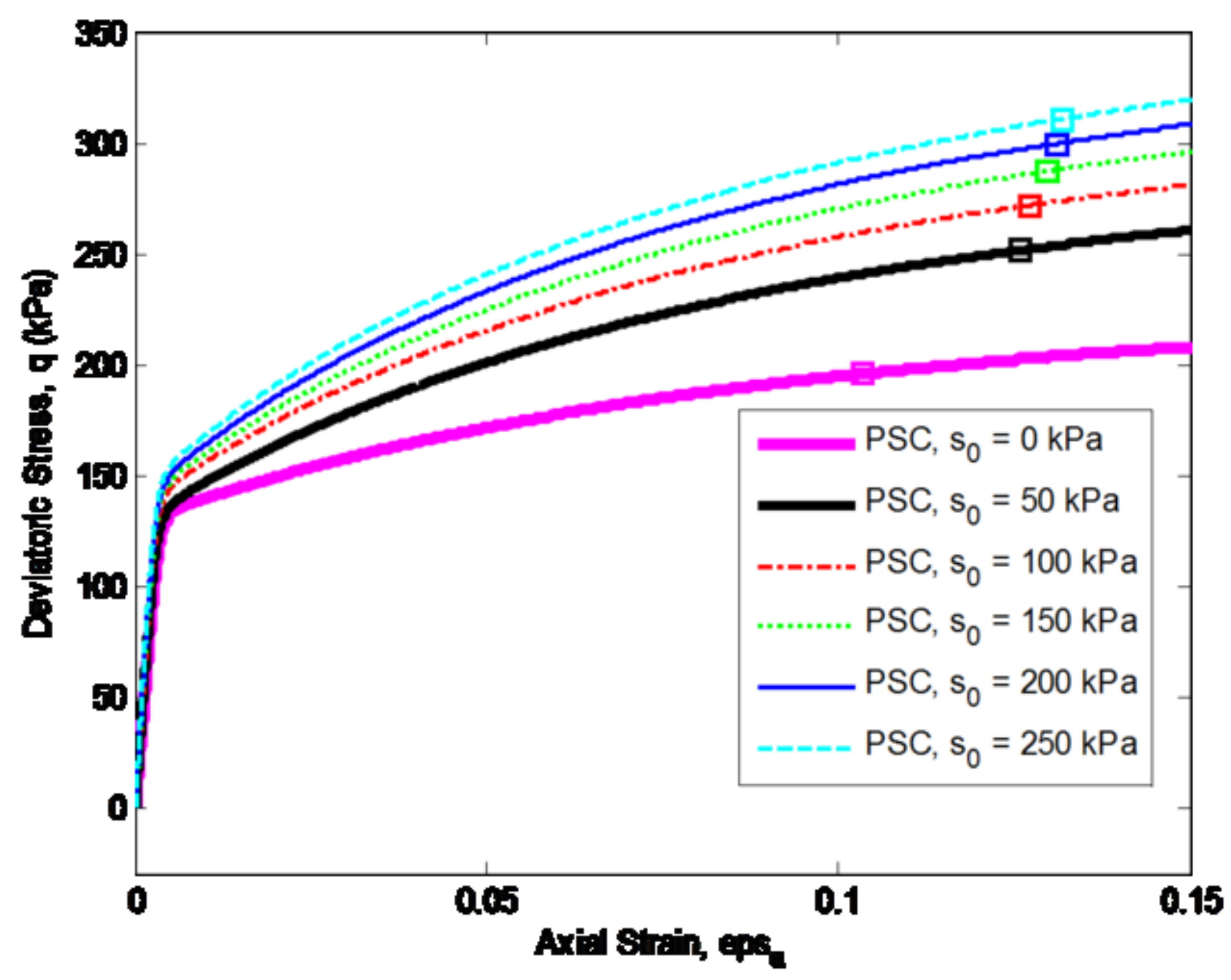


Figure 5. Prediction of onset of strain localization for PSC tests under different drainage conditions (pnet, $0=100 \mathrm{kPa}$, initial suction $\mathrm{s}_{0}=50 \mathrm{kPa}$ ): a) Stress-strain response

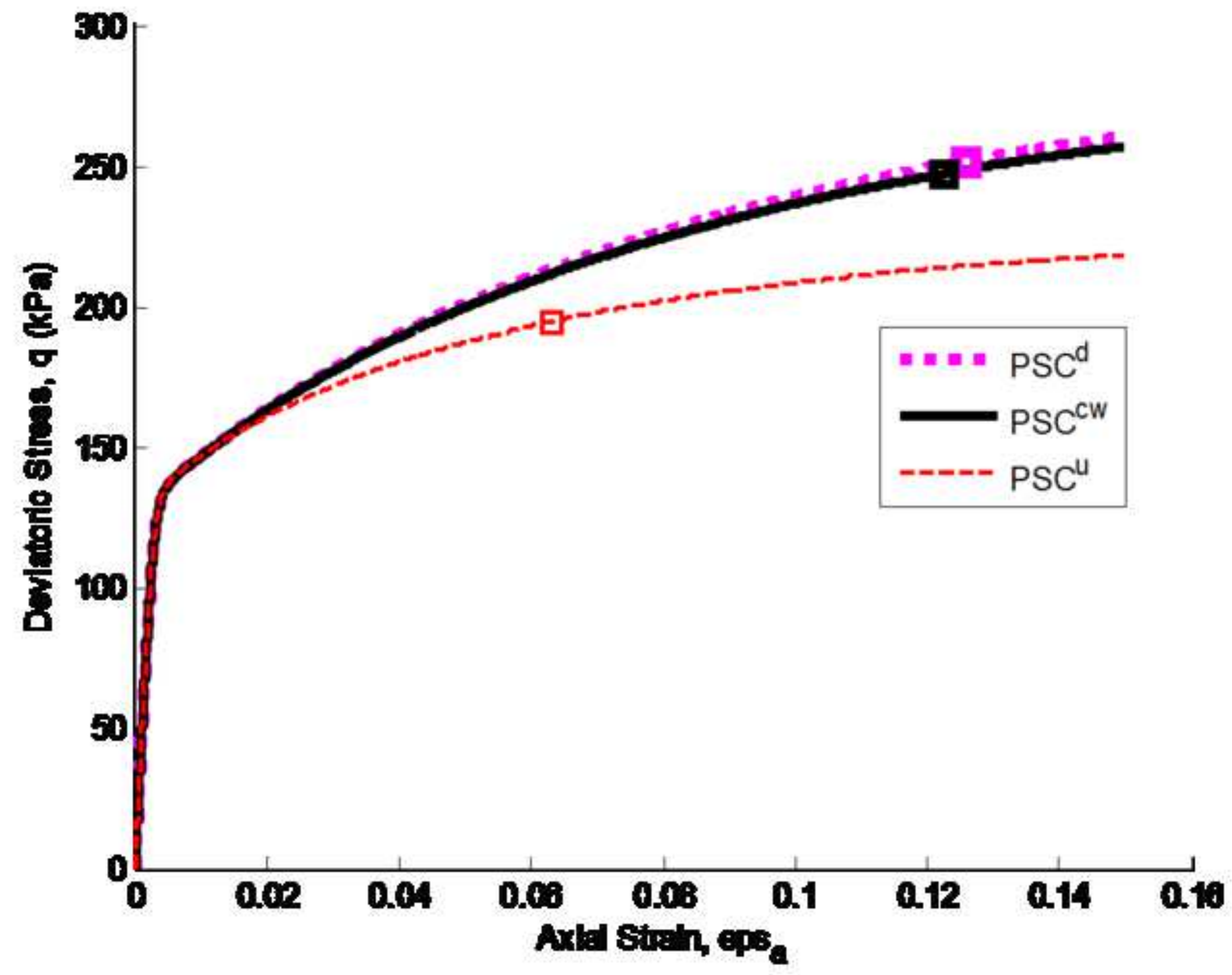


Figure 5. Prediction of onset of strain localization for PSC tests under different drainage conditions $\left(p_{n e t, 0}=100 \mathrm{kPa}\right.$, initial suction $\left.s_{0}=50 \mathrm{kPa}\right)$ : b) Strain-strain response

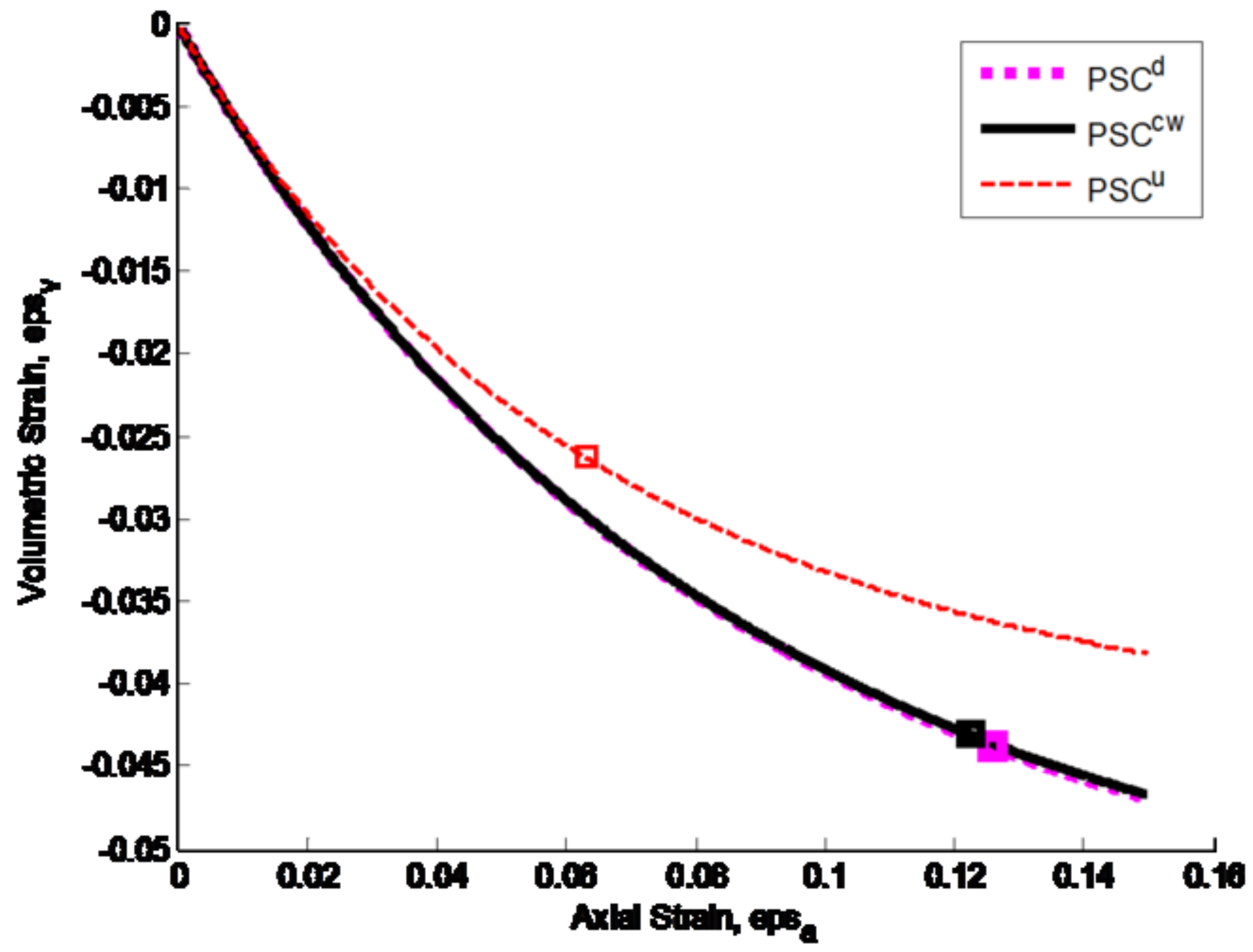


Figure 5. Prediction of onset of strain localization for PSC tests under different drainage conditions (pnet, $0=100 \mathrm{kPa}$, initial suction $\left.\mathrm{s}_{0}=50 \mathrm{kPa}\right)$ : c) Suction-strain response

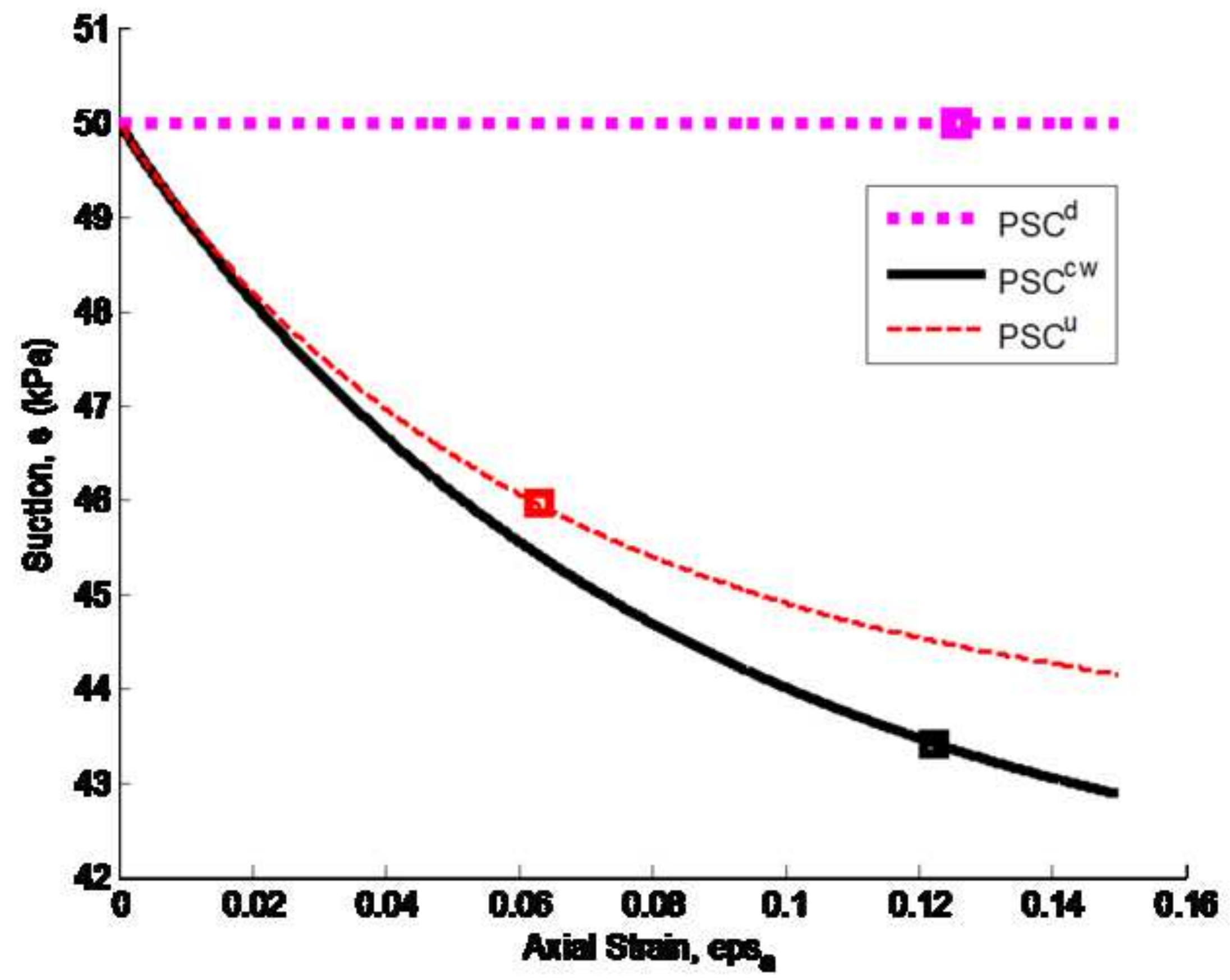


Figure 6. Critical axial strain versus net mean stress at onset of strain localization for PSC tests:

a) Drained tests

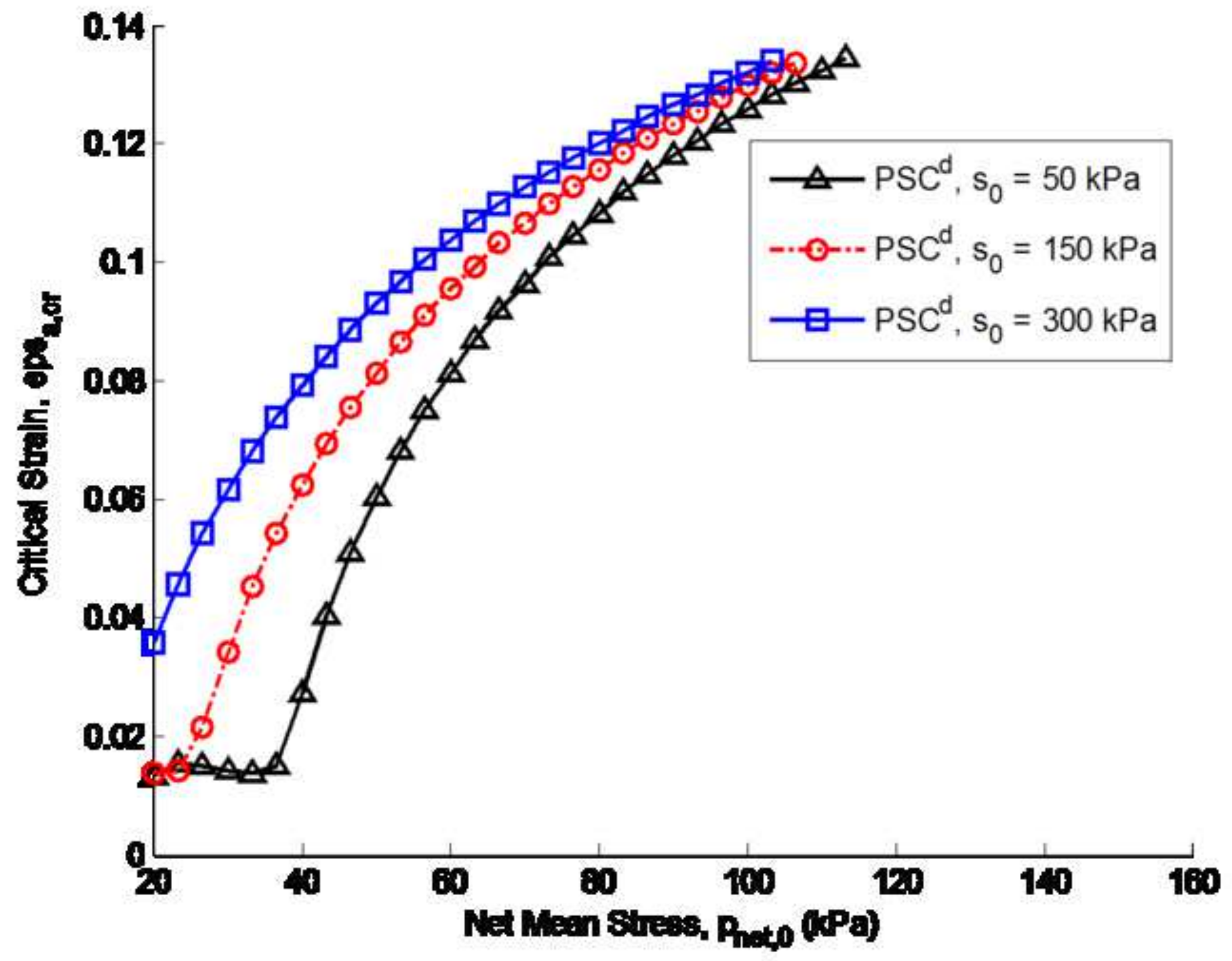


Figure 6. Critical axial strain versus net mean stress at onset of strain localization for PSC tests: b) Constant water content tests

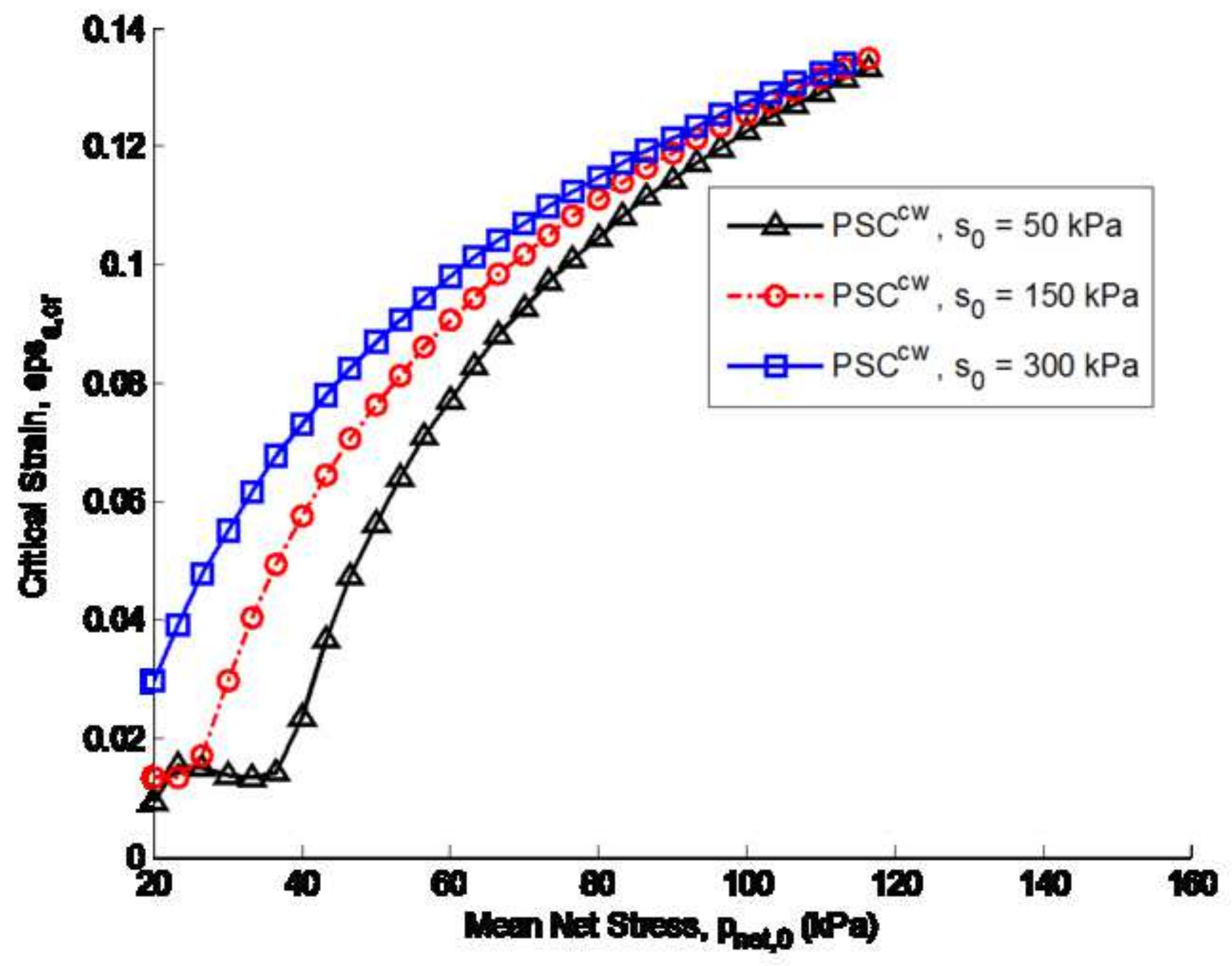


Figure 6. Critical axial strain versus net mean stress at onset of strain localization for PSC tests:

c) Undrained tests

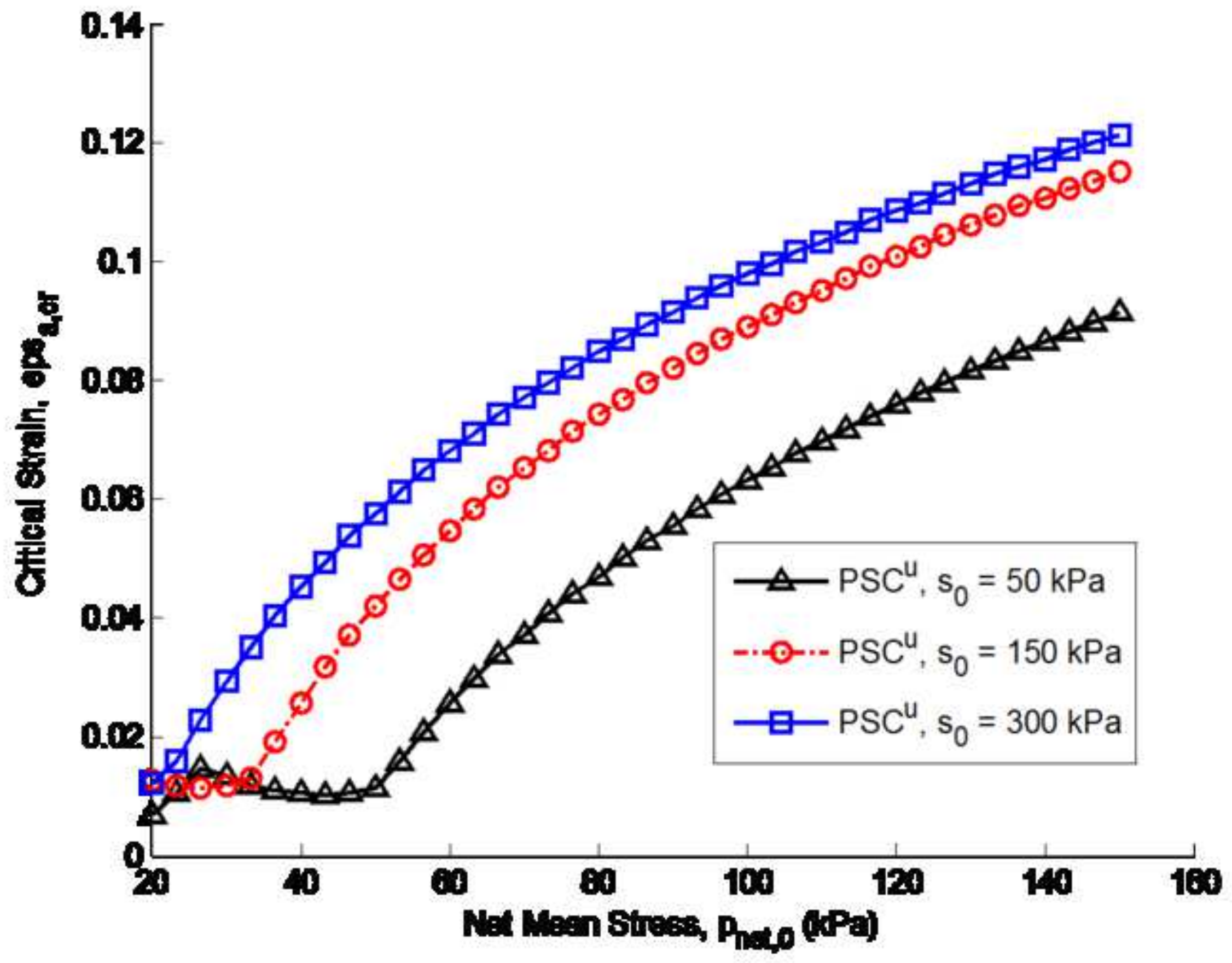


Figure 7. Deformation band orientation at onset of strain localization for PSC tests: a ) Drained tests

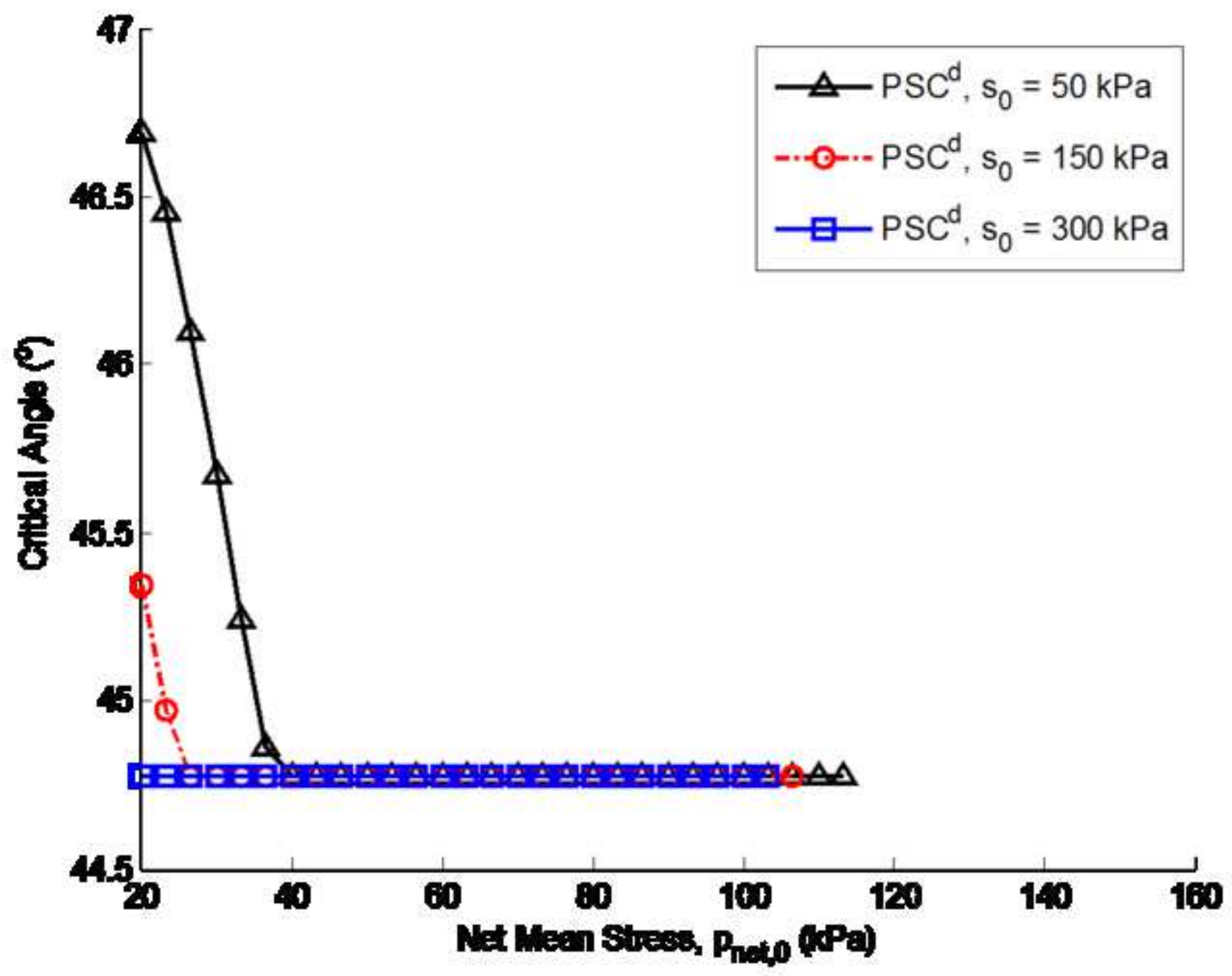


Figure 7. Deformation band orientation at onset of strain localization for PSC tests:

b) Constant water content tests

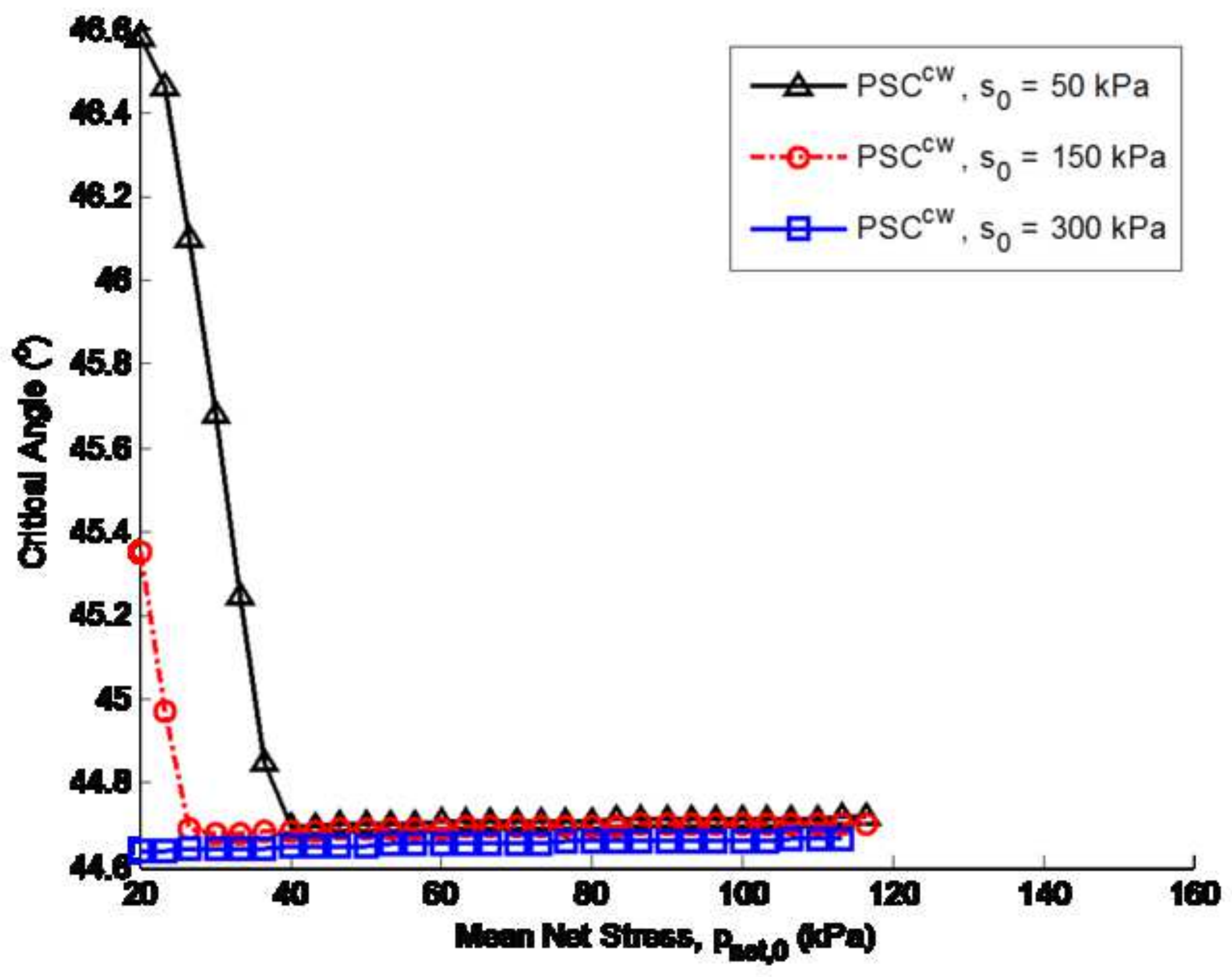


Figure 7. Deformation band orientation at onset of strain localization for PSC tests: c) Undrained tests

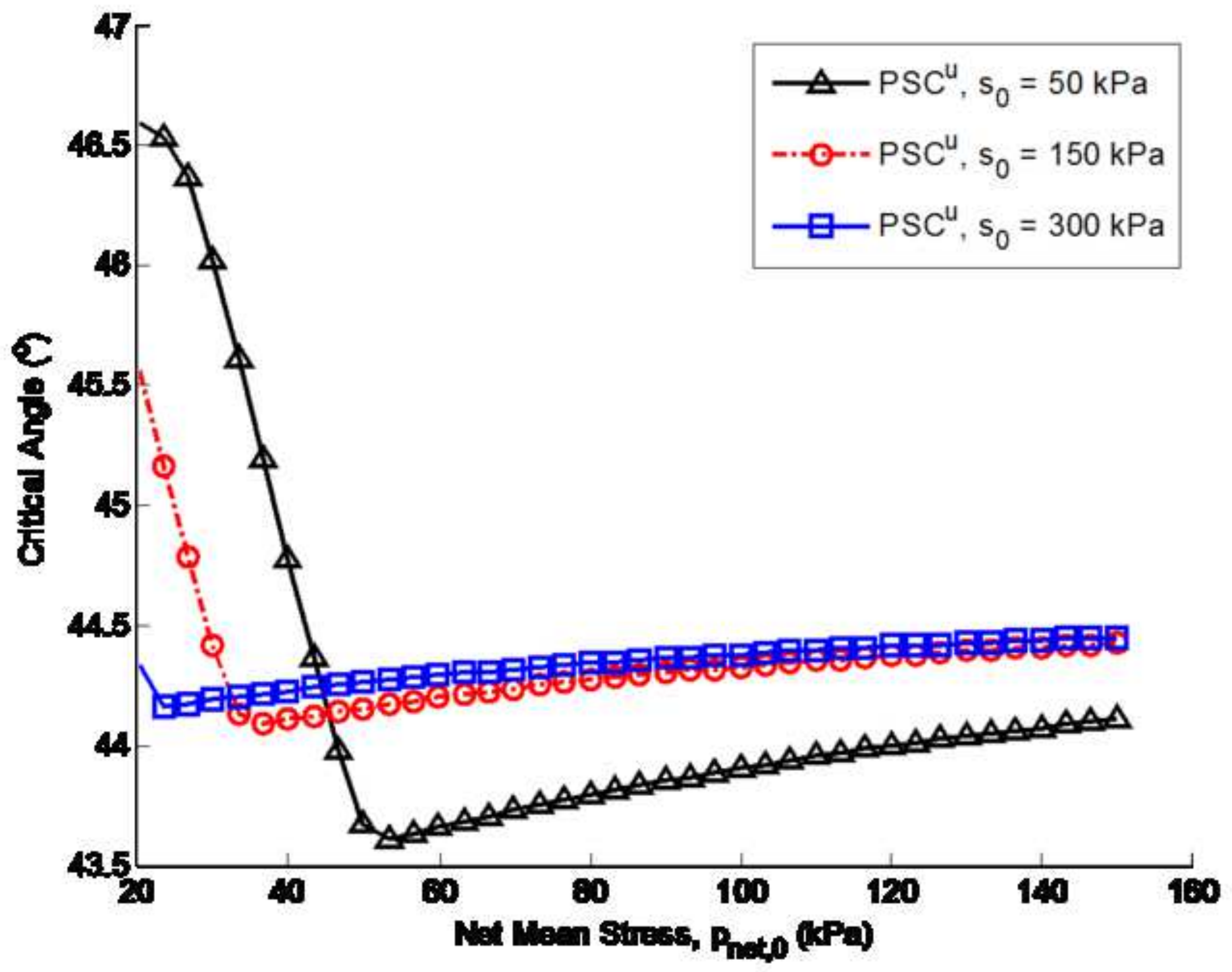


Figure 8. Eigenvector angle versus net stress for PSC tests: a) Drained tests

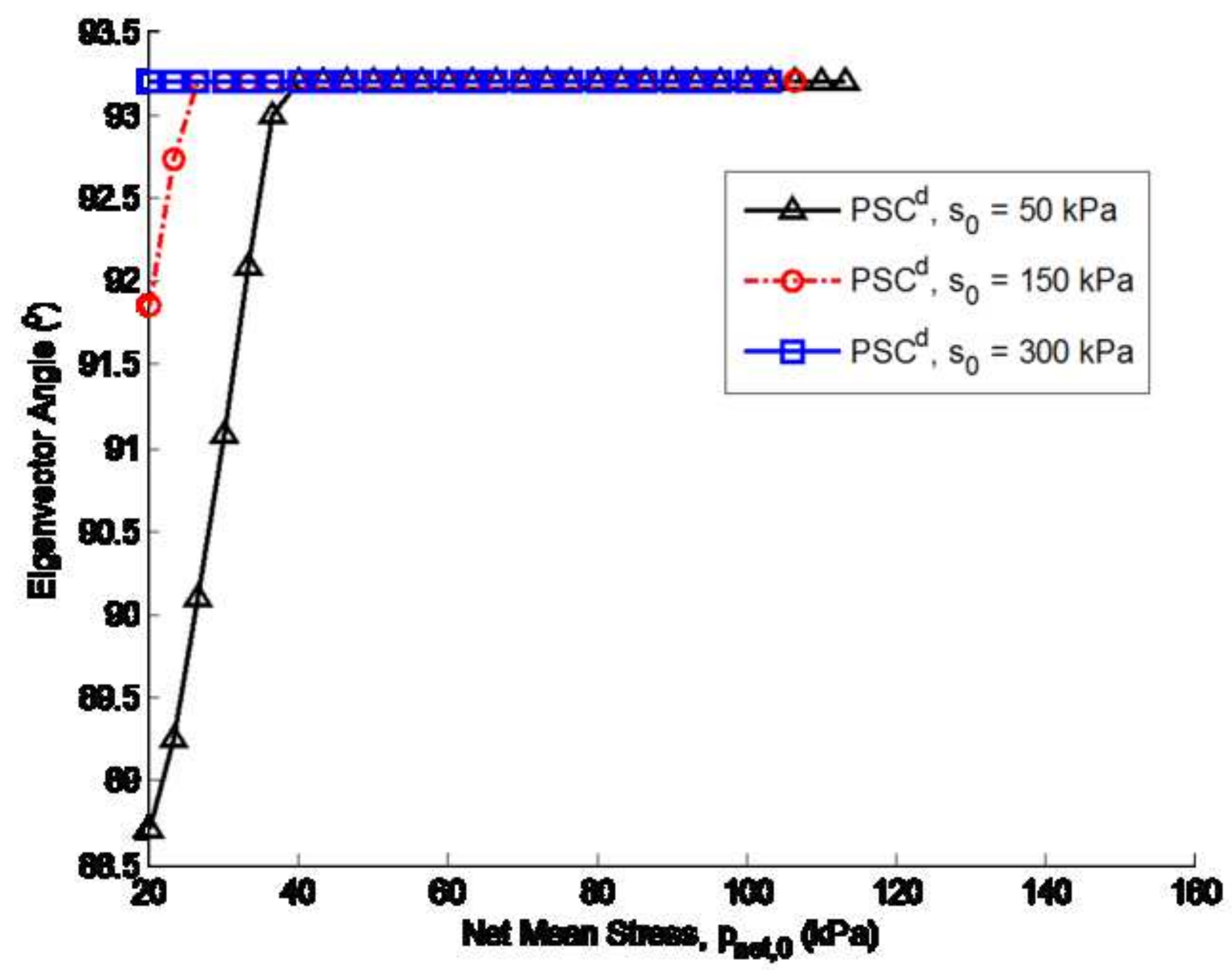


Figure 8. Eigenvector angle versus net stress for PSC tests: b) Constant water content tests

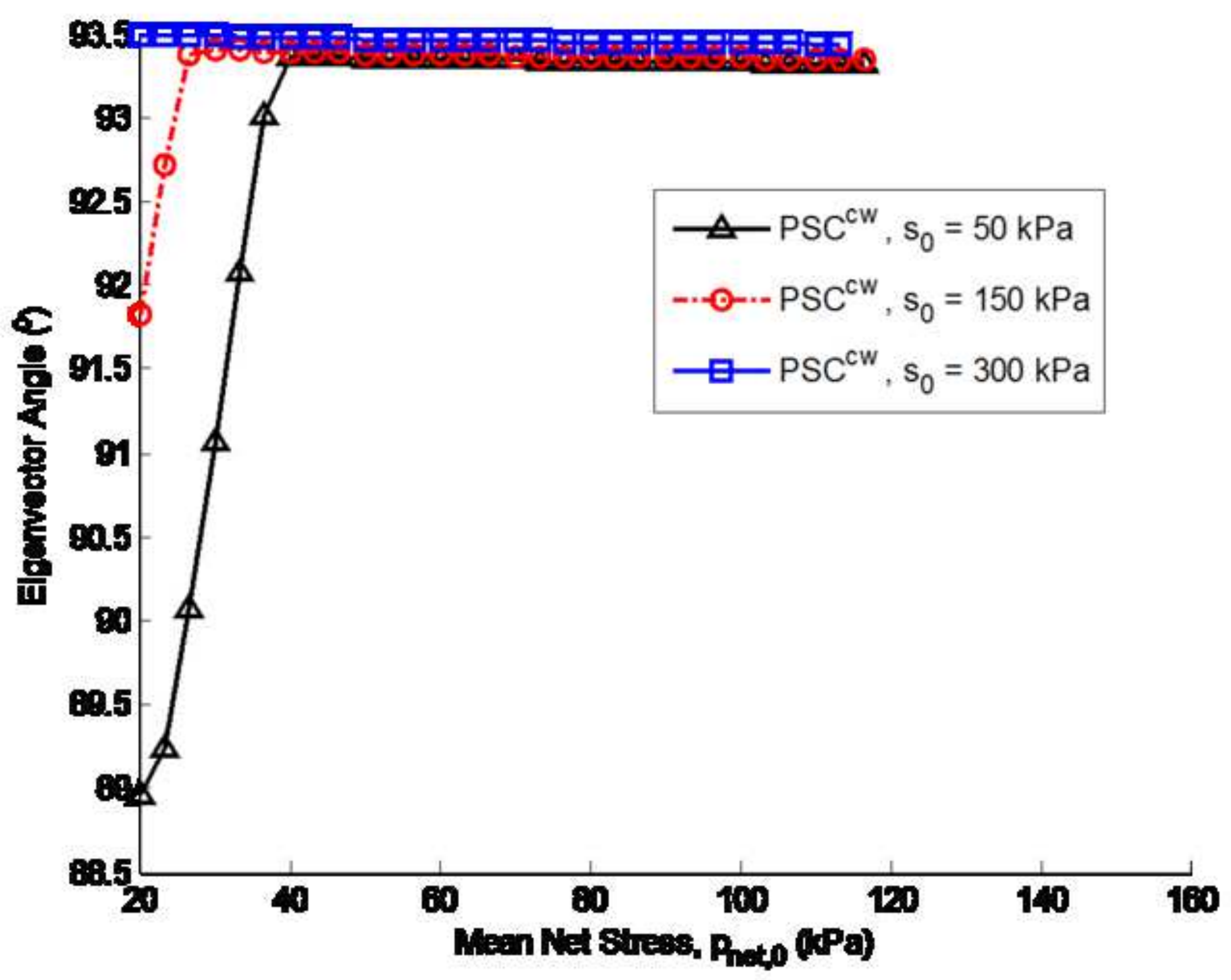


Figure 8. Eigenvector angle versus net stress for PSC tests: c) Undrained tests

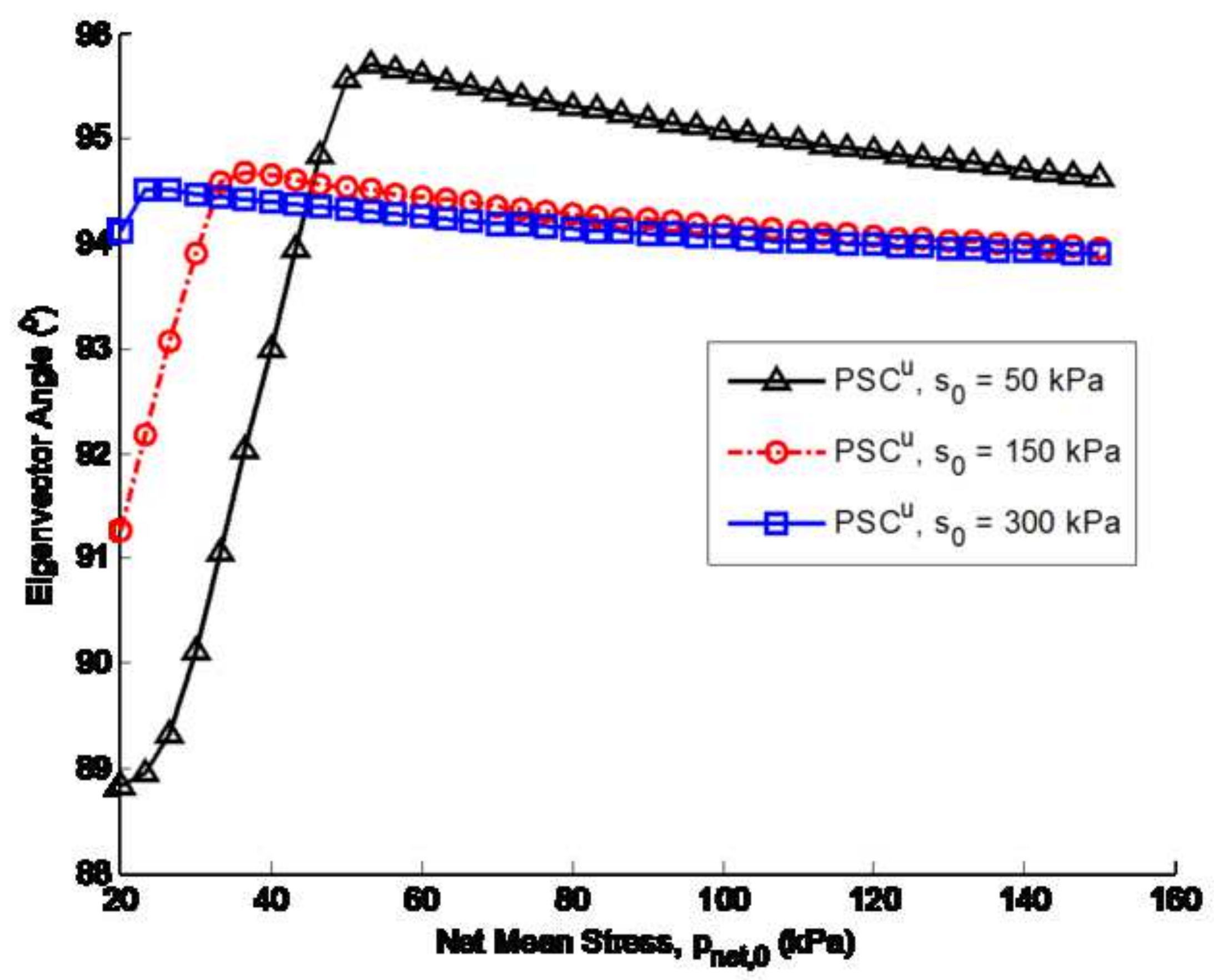

